\title{
Cryptanalysis of CLT13 Multilinear Maps with Independent Slots
}

\author{
Jean-Sébastien Coron, Luca Notarnicola \\ University of Luxembourg \\ jean-sebastien.coron@uni.lu, luca.notarnicola@uni.lu
}

\begin{abstract}
Many constructions based on multilinear maps require independent slots in the plaintext, so that multiple computations can be performed in parallel over the slots. Such constructions are usually based on CLT13 multilinear maps, since CLT13 inherently provides a composite encoding space, with a plaintext ring $\bigoplus_{i=1}^{n} \mathbb{Z} / g_{i} \mathbb{Z}$ for small primes $g_{i}$ 's. However, a vulnerability was identified at Crypto 2014 by Gentry, Lewko and Waters, with a lattice-based attack in dimension 2, and the authors have suggested a simple countermeasure. In this paper, we identify an attack based on higher dimension lattice reduction that breaks the author's countermeasure for a wide range of parameters. Combined with the Cheon et al. attack from Eurocrypt 2015, this leads to the recovery of all the secret parameters of CLT13, assuming that low-level encodings of almost zero plaintexts are available. We show how to apply our attack against various constructions based on composite-order CLT13. For the [FRS17] construction, our attack enables to recover the secret CLT13 plaintext ring for a certain range of parameters; however, breaking the indistinguishability of the branching program remains an open problem.
\end{abstract}

\section{Introduction}

Multilinear maps. In 2013, Garg, Gentry and Halevi described the first plausible construction of cryptographic multilinear maps based on ideal lattices [GGH13a]. Since then many amazing applications of multilinear maps have been found in cryptography, including program obfuscation $\left[\mathrm{GGH}^{+} 13 \mathrm{~b}\right]$. Shortly after the publication of GGH13, an analogous construction over the integers was described in [CLT13], based on the DGHV fully homomorphic encryption scheme [DGHV10]. The GGH15 scheme is the third known family of multilinear maps, based on the LWE problem with encoding over matrices [GGH15].

In the last few years, many attacks have appeared against multilinear maps, and the security of multilinear maps is still poorly understood. An important class of attacks against multilinear maps are "zeroizing attacks", which can recover the secret parameters from encodings of zero, using linear algebra. For the non-interactive multipartite Diffie-Hellman key exchange, the zeroizing attack from Cheon et al. $\left[\mathrm{CHL}^{+} 15\right]$ recovers all secret parameters from CLT13; the attack can also be extended to encoding variants where encodings of zero are not directly available $\left[\mathrm{CGH}^{+} 15\right]$. The zeroizing attack from [HJ16] also breaks the Diffie-Hellman key-exchange over GGH13. Finally, the key exchange over GGH15 was also broken in [CLLT16], using an extension of the Cheon et al. zeroizing attack.

Even though direct multipartite key exchange protocols are broken for the three known families of multilinear maps, more complex constructions based on multilinear maps are not necessarily broken, in particular indistinguishability obfuscation (iO); namely low-level encodings of zero are generally not available in $\mathrm{iO}$ constructions. However the Cheon et al. attack against CLT13 was extended in $\left[\mathrm{CGH}^{+} 15\right]$ to matrix branching programs where the input can be partitioned into three independent sets. The attack was further extended in [CLLT17] to branching programs without a simple input partition structure, using a tensoring technique. For GGH13 based obfuscation, Miles, Sahai and Zhandry introduced "annihilation attacks" that can break a certain class of matrix branching programs [MSZ16]; the attack was later extended in [CGH17] to break the [GGH ${ }^{+} 13 \mathrm{~b}$ ] obfuscation under GGH13, using a variant of the input partitioning attack. Finally, Chen, Vaikuntanathan and Wee described in [CVW18] an attack against iO over GGH15, based on computing the rank of a well chosen matrix. In general, the above attacks only apply against branching programs with a simple structure, and breaking more complex constructions (such as dual-input branching programs) is currently infeasible. 
Multilinear maps with independent slots. Many constructions based on multilinear maps require independent slots in the plaintext, so that multiple computations can be performed in parallel over the slots when evaluating the multilinear map. For example, [GLW14] and [GLSW15] use independent slots to obtain improved security reductions for witness encryption and obfuscation. Multilinear maps with independent slots were also used in the circuit based constructions of [AB15,Zim15]. The construction from [FRS17], which gives a powerful technique for preventing zeroizing attacks against $\mathrm{iO}$, is also based on multilinear maps with independent slots.

The CLT13 multilinear map scheme inherently supports a composite integer encoding space, with a plaintext ring $\mathbb{Z} / G \mathbb{Z} \simeq \bigoplus_{i=1}^{n} \mathbb{Z} / g_{i} \mathbb{Z}$ for small secret primes $g_{i}$ 's and $G=g_{1} \cdots g_{n}$. For example, in the construction from [FRS17], every branching program works independently modulo each $g_{i}$. In that case, the main difference with the original CLT13 is that the attacker can obtain encodings of subring elements which are zero modulo all $g_{i}$ 's except one; for example, in [FRS17] this would be done by carefully choosing the input so that all branching programs would evaluate to zero except one. Whereas in the original CLT13 construction, one never provides encodings of subring elements; instead one uses an "all-or-nothing" approach: either the plaintext element is zero modulo all $g_{i}$ 's, or it is non-zero modulo all $g_{i}$ 's (with high probability).

The attack and countermeasure from [GLW14]. At Crypto 2014, Gentry, Lewko and Waters observed that using CLT13 with independent slots leads to a simple lattice attack in dimension 2, which efficiently recovers the (secret) plaintext ring $\bigoplus_{i=1}^{n} \mathbb{Z} / g_{i} \mathbb{Z}$ [GLW14, Appendix B]. Namely, when using CLT13 with independent slots, the attacker can obtain encodings where all slots are zero modulo $g_{i}$ except one. For example, for a matrix branching program evaluation as in [FRS17], the result of the program evaluation could have the form:

$$
A(x) \equiv \sum_{i=1}^{n} h_{i} \cdot\left(r_{i}+m_{i} \cdot\left(g_{i}^{-1} \bmod p_{i}\right)\right) \cdot \frac{x_{0}}{p_{i}}\left(\bmod x_{0}\right)
$$

where $m_{i}=0$ for all $i$ except $m_{j} \neq 0$ for some $1 \leq j \leq n$. This implies:

$$
g_{j} \cdot A(x) \equiv h_{j}\left(r_{j} g_{j}+m_{j}\right) \frac{x_{0}}{p_{j}}+\sum_{i \neq j} g_{j} h_{i} r_{i} \frac{x_{0}}{p_{i}} \quad\left(\bmod x_{0}\right)
$$

and therefore $g_{j} \cdot A(x) \bmod x_{0}$ is "small" (significantly smaller than $x_{0}$ ). Since $g_{j}$ is very small, we can then recover $g_{j}$ using lattice reduction in dimension 2, while normally the $g_{i}$ 's are secret in CLT13. Moreover, once we know $g_{j}$, we can simply multiply the evaluation by $g_{j}$ to obtain a "small" result, even if the evaluation of the branching program is non-zero modulo $g_{j}$; in particular, this cancels the effect of the protection against input partitioning from [FRS17].

The countermeasure considered in [GLW14, Appendix B] is to give many "buddies" to each $g_{i}$, so that we do not have a plaintext element which is non-zero modulo a single isolated $g_{i}$. Then, either an encoding is 0 modulo $g_{i}$ and all its prime buddies $g_{j}$, or it is (with high probability) non-zero modulo all of them. In other words, instead of using individual $g_{i}$ 's to define the plaintext slots, every slot is defined modulo a product of $\theta$ prime $g_{i}$ 's, for some $1 \leq \theta<n$. Therefore, we obtain a total of $\lfloor n / \theta\rfloor$ plaintext slots (instead of $n$ ). While the above attack can be extended by multiplying $A(x)$ by the $\theta$ corresponding $g_{i}$ 's, for large enough $\theta$ the right-hand side of the equation is not "small" anymore and the attack is thwarted.

Our contributions. In this paper we identify an attack based on higher dimension lattice reduction that breaks the countermeasure from [GLW14, Appendix B] for a wide range of parameters, with significant impact on the security of CLT13 multilinear maps with independent slots. More precisely, our contributions are as follows: 
1. Analysis of the attack from [GLW14]. Our first contribution is to provide a theoretical study of the above attack, in order to derive a precise bound on $\theta$ as a function of the CLT13 parameters (there was no explicit bound in [GLW14]), where $\theta$ is the number of primes $g_{i}$ 's for each plaintext slot. We argue that, when $\nu$ denotes the number of bits that can be extracted from zero-testing in CLT13, the 2-dimensional lattice attack requires:

$$
\alpha \theta<\frac{\nu}{2}
$$

where $\alpha$ is the bit size of the $g_{i}$ 's.

2. Breaking the countermeasure from [GLW14]. Our main contribution is to extend the 2dimensional attack to break the countermeasure for larger values of $\theta$. Our attack is based on higher dimension lattice reduction, by using a similar orthogonal lattice attack as in [NS99] for solving the hidden subset sum problem. In this extension, we use $\ell$ encodings $\left\{c_{j}: 1 \leq j \leq \ell\right\}$ where the corresponding plaintexts have only $\theta$ non-zero components modulo the $g_{i}$ 's (instead of $\ell=1$ in the previous attack). Using a lattice attack in dimension $\ell+1$, we show that our attack requires the approximate condition $\left(1+\frac{1}{\ell}\right) \alpha \theta<\nu$ for the parameters. Therefore, for moderately large values of $\ell$, we get the simpler condition:

$$
\alpha \theta<\nu
$$

which improves (1) by a factor 2 .

In the same vein, we show how to further improve this condition by considering products of encodings of the form $c_{j} \cdot d_{k}$ for $1 \leq j \leq \ell$ and $1 \leq k \leq d$, where as previously, the plaintexts of the $c_{j}$ 's have only $\theta$ non-zero components modulo the $g_{i}$ 's. In that case, using a variant of the previous lattice attack (this time in dimension $\ell+d$ ), the bound improves to:

$$
\alpha \theta=\mathcal{O}\left(\nu^{2}\right)
$$

The above bound also applies when a vector of zero-testing elements is available, instead of a single $p_{z t}$. While the original attack from [GLW14] recovers the secret plaintext ring of CLT13, we additionally recover the plaintext messages $\left\{m_{j}: 1 \leq j \leq \ell\right\}$ for the encodings $\left\{c_{j}: 1 \leq j \leq \ell\right\}$, up to a scaling factor.

We provide in Section 4.5 the result of practical experiments. For the original parameters of [CLT13], our attack takes a few seconds for $\theta=40$, and a few hours for $\theta$ as large as 160, while the original attack from [GLW14] only works for $\theta=1$. In summary, our attack is more powerful than the attack in [GLW14], as it additionally recovers secret information about the plaintext messages, moreover for much larger values of $\theta$. Finally, we suggest a set of secure parameters for CLT13 multilinear maps that prevents our extended attack. For $\lambda=80$ bits of security, we recommend to take $\theta \geq 1789$.

3. Recovering all the secret parameters of CLT13. For the range of parameters derived previously, we show how to combine our attack with the Cheon et al. attack from $\left[\mathrm{CHL}^{+} 15\right]$, in order to recover all secret parameters of CLT13. More precisely, when intermediate-level encodings of partially zero messages are available, our approach consists in applying the lattice attack to generate intermediate-level encodings of zero; then the Cheon et al. attack is applied on these newly-created encodings of zero, to recover all secret parameters.

4. Application to CLT13-based constructions. Finally we show how our attack affects the parameter selection of several schemes based on CLT13 multilinear maps with independent slots, namely the constructions from [GLW14,GLSW15,Zim15] and [FRS17]. For the [FRS17] construction, our attack enables to recover the secret CLT13 plaintext ring for a certain range of parameters; however, breaking the indistinguishability of the branching program remains an open problem. 
Source code. We provide in

https://pastebin. com/7WEMHBE9

the source code of our attacks in Sage $\left[\mathrm{S}^{+} 17\right]$.

\section{The CLT13 Multilinear Map Scheme}

We first recall the CLT13 multilinear map scheme over the integers [CLT13]. For $n \in \mathbb{Z}_{\geq 1}$, the instance generation of CLT13 generates $n$ distinct secret "large" primes $p_{1}, \ldots, p_{n}$ of size $\eta$ bits, and publishes the modulus $x_{0}=\prod_{i=1}^{n} p_{i}$. We let $\gamma$ denote the bit size of $x_{0}$; therefore $\gamma \simeq n \cdot \eta$. One also generates $n$ distinct secret "small" prime numbers $g_{1}, \ldots, g_{n}$ of size $\alpha$ bits. The plaintext ring is composite, i.e. a plaintext is an element $\boldsymbol{m}=\left(m_{1}, \ldots, m_{n}\right)$ of the $\operatorname{ring} \mathbb{Z} / G \mathbb{Z} \simeq \bigoplus_{i=1}^{n} \mathbb{Z} / g_{i} \mathbb{Z}$ where $G=\prod_{i=1}^{n} g_{i}$. Let $\kappa \in \mathbb{Z}_{\geq 1}$ be the multilinearity parameter. For $k \in\{1, \ldots, \kappa\}$, an encoding at level $k$ of the plaintext $\boldsymbol{m}$ is an integer $c \in \mathbb{Z}$ such that

$$
c \equiv \frac{r_{i} g_{i}+m_{i}}{z^{k}} \quad\left(\bmod p_{i}\right), \text { for all } 1 \leq i \leq n
$$

for "small" random integers $r_{i}$ of bit size $\rho$. The random mask $z \in\left(\mathbb{Z} / x_{0} \mathbb{Z}\right)^{\times}$is the same for all encodings. It is clear that two encodings at the same level can be added, and the underlying plaintexts get added in the ring $\mathbb{Z} / G \mathbb{Z}$. Similarly, the product of two encodings at level $i$ and $j$ gives an encoding of the product plaintexts at level $i+j$, as long as the numerators in (2) do not grow too large, i.e. they must remain smaller than each $p_{i}$.

For an encoding at the last level $\kappa$, one defines the following zero-testing procedure. The instance generation publishes the zero-testing parameter $p_{z t}$, defined by

$$
p_{z t}=\sum_{i=1}^{n} h_{i} z^{\kappa}\left(g_{i}^{-1} \bmod p_{i}\right) \frac{x_{0}}{p_{i}} \bmod x_{0}
$$

where $h_{i} \in \mathbb{Z}$ are "small" random integers of size $n_{h}$ bits. Given an encoding $c$ at the last level $\kappa$, we compute the integer:

$$
\omega:=p_{z t} \cdot c \bmod x_{0} \equiv \sum_{i=1}^{n} h_{i}\left(r_{i}+m_{i}\left(g_{i}^{-1} \bmod p_{i}\right)\right) \frac{x_{0}}{p_{i}} \quad\left(\bmod x_{0}\right)
$$

and we consider that $c$ encodes the zero message if $\omega$ is "small" compared to $x_{0}$. Namely, if $m_{i}=0$ for all $i$, we obtain $\omega \equiv \sum_{i=1}^{n} h_{i} r_{i} \frac{x_{0}}{p_{i}}\left(\bmod x_{0}\right)$, and since the integers $h_{i}$ and $r_{i}$ are "small", the resulting $\omega$ will be "small" compared to $x_{0}$.

More precisely, let $\rho_{f}$ be the maximum bit size of the noise $r_{i}$ in the encodings. Then the integers $h_{i} r_{i} x_{0} / p_{i}$ have size roughly $\gamma-\eta+n_{h}+\rho_{f}$, and therefore letting

$$
\nu=\eta-n_{h}-\rho_{f},
$$

the integers $h_{i} r_{i} x_{0} / p_{i}$ have size roughly $\gamma-\nu$ bits. Therefore, when $m_{i}=0$ for all $i$, the integer $\omega$ has size roughly $\gamma-\nu$ bits; whereas when $m_{i} \neq 0$ for some $i$, we expect that $\omega$ is of full size modulo $x_{0}$, that is $\gamma$ bits. The parameter $\nu$ in (5) corresponds to the number of bits that can be extracted from zero-testing; namely from (4), the $\nu$ most significant bits of $\omega$ only depend on the plaintext messages $m_{i}$, and not on the noise $r_{i}$. Note that to get a proper zero-testing procedure, one needs to use a vector of $n$ elements $p_{z t}$; namely with a single $p_{z t}$ there exist encodings $c$ with $m_{i} \neq 0$ while $p_{z t} \cdot c$ is "small" modulo $x_{0}$. In the rest of the paper, for simplicity, we mainly consider a single $p_{z t}$, as it is usually the case in constructions over CLT13 multilinear maps. We refer to [CLT13, Section 3.1] for the setting of the parameters. 


\section{Basic Attack against CLT13 with Independent Slots}

Many constructions based on multilinear maps require independent slots in the plaintext, so that multiple computations can be performed in parallel over the slots when evaluating the multilinear map; see for example [GLW14,GLSW15] and [AB15,Zim15,FRS17]. The CLT13 multilinear maps inherently provide independent slots, as the plaintext ring is $\bigoplus_{i=1}^{n} \mathbb{Z} / g_{i} \mathbb{Z}$ for small secret primes $g_{1}, \ldots, g_{n}$. Therefore we can have independent computations performed over the $n$ plaintext slots modulo $g_{i}$; for example, in the construction from [FRS17], every branching program works independently modulo each $g_{i}$.

The basic attack from [GLW14]. When using CLT13 with independent slots, the attacker can obtain encodings of plaintext elements where all slots are zero modulo $g_{i}$ except one. For example, in the [FRS17] construction where each branching program works modulo $g_{i}$, the attacker can choose the input so that the resulting evaluation is 0 modulo all $g_{i}$ 's except one, say $g_{1}$, without loss of generality. Let $c$ be a level- $\kappa$ encoding of a plaintext $\boldsymbol{m}=\left(m_{1}, \ldots, m_{n}\right)$ where $m_{i}=0$ for all $2 \leq i \leq n$. From Equation (4) we obtain the following zero-testing evaluation:

$$
\omega \equiv h_{1} \cdot m_{1} \cdot\left(g_{1}^{-1} \bmod p_{1}\right) \cdot \frac{x_{0}}{p_{1}}+\sum_{i=1}^{n} h_{i} \cdot r_{i} \cdot \frac{x_{0}}{p_{i}} \quad\left(\bmod x_{0}\right)
$$

This implies:

$$
g_{1} \cdot \omega \equiv h_{1} \cdot m_{1} \cdot \frac{x_{0}}{p_{1}}+\sum_{i=1}^{n} g_{1} \cdot h_{i} \cdot r_{i} \cdot \frac{x_{0}}{p_{i}} \quad\left(\bmod x_{0}\right)
$$

and therefore $g_{1} \cdot \omega \bmod x_{0}$ is significantly smaller than $x_{0}$, as the integers $h_{i}$ and $r_{i}$ are "small". This implies that we can recover $g_{1}$, and similarly the other $g_{i}$ 's using lattice reduction in dimension 2, while normally the $g_{i}$ 's are secret in CLT13. This eventually recovers the plaintext ring. We analyze the attack below.

The countermeasure from [GLW14]. The following countermeasure was therefore suggested by the authors: instead of using individual $g_{i}$ 's to define the plaintext slots, every slot is defined modulo a product of $\theta$ prime $g_{i}$ 's, where $2 \leq \theta<n$. Therefore, a plaintext element cannot be non-zero modulo a single prime $g_{i}$; it has to be non-zero modulo at least $\theta$ primes $g_{i}$ 's. This gives a total of $n / \theta$ plaintext slots (instead of $n$ ); for simplicity we assume that $\theta$ divides $n$.

Therefore, the original plaintext ring $R=\mathbb{Z} / g_{1} \mathbb{Z} \times \cdots \times \mathbb{Z} / g_{n} \mathbb{Z}$ can be rewritten as $R=\bigoplus_{j=1}^{n / \theta} R_{j}$, where for all $1 \leq j \leq n / \theta$, the subrings $R_{j}$ are such that $R_{j} \simeq \bigoplus_{i=1}^{\theta} \mathbb{Z} / g_{(j-1) \theta+i} \mathbb{Z}$. We can assume that the attacker can obtain encodings of random subring plaintexts in $R_{j}$ for any $1 \leq j \leq n / \theta$. In that case, the attacker obtains an encoding $c$ of $\boldsymbol{m}=\left(m_{1}, \ldots, m_{n}\right) \in R$ where $m_{i} \equiv 0\left(\bmod g_{i}\right)$ for all $i \in\{1, \ldots, n\} \backslash\{(j-1) \theta+1, \ldots, j \theta\}$. In that case we will say that $\boldsymbol{m}$ has non-zero support of length $\theta$.

Analysis of the basic attack. In this section we analyze in more details the attack from [GLW14], and we derive an explicit bound on the parameter $\theta$, as a function of the other CLT13 parameters. Given an integer $1 \leq \theta<n$ (the above attack is obtained for $\theta=1$ ), we consider a message having non-zero support of length $\theta$; that is, (without loss of generality) of the form $\boldsymbol{m}=\left(m_{1}, \ldots, m_{n}\right) \in \mathbb{Z}^{n}$ with $0 \leq m_{i}<g_{i}$ such that $m_{i}=0$ for $\theta+1 \leq i \leq n$, i.e. we assume that the non-zero support of $\boldsymbol{m}$ is located in the first slot. We consider a top level $\kappa$ encoding $c$ of $\boldsymbol{m}$, that is:

$$
c \equiv \frac{r_{i} g_{i}+m_{i}}{z^{\kappa}} \quad\left(\bmod p_{i}\right), \quad 1 \leq i \leq n
$$


with integers $r_{i}$ of bit size $\rho_{f}$. From zero-testing, we obtain from (4):

$$
\omega \equiv p_{z t} \cdot c \equiv \sum_{i=1}^{\theta} h_{i}\left(g_{i}^{-1} \bmod p_{i}\right) m_{i} \frac{x_{0}}{p_{i}}+\sum_{i=1}^{n} h_{i} r_{i} \frac{x_{0}}{p_{i}} \quad\left(\bmod x_{0}\right)
$$

By multiplying out by $g:=\prod_{i=1}^{\theta} g_{i}$ we obtain

$$
\begin{aligned}
g \omega & \equiv \sum_{i=1}^{\theta} h_{i} m_{i} \frac{g}{g_{i}} \frac{x_{0}}{p_{i}}+\sum_{i=1}^{n} g h_{i} r_{i} \frac{x_{0}}{p_{i}} \quad\left(\bmod x_{0}\right), \\
g \omega & \equiv U \quad\left(\bmod x_{0}\right)
\end{aligned}
$$

where $U=\sum_{i=1}^{\theta} h_{i} m_{i}\left(g / g_{i}\right)\left(x_{0} / p_{i}\right)+\sum_{i=1}^{n} g h_{i} r_{i}\left(x_{0} / p_{i}\right)$. Since the integers $h_{i}$ and $r_{i}$ are "small" in order to ensure correct zero-testing, the integer $U$ is "small" in comparison to $x_{0}$. More precisely, the proposition below shows that if $g \cdot U$ is a bit smaller than $x_{0}$, then we can recover $g$ and $U$ by lattice reduction in dimension 2 .

Proposition 1. Let $g, \omega, U \in \mathbb{Z}_{\geq 1}$ and $x_{0} \in \mathbb{Z}_{\geq 1}$ be such that $g \omega \equiv U\left(\bmod x_{0}\right), \omega \in\left(\mathbb{Z} / x_{0} \mathbb{Z}\right)^{\times}$ and $\operatorname{gcd}(U, g)=1$. Assume that $g \cdot U<x_{0} / 10$. Given $\omega$ and $x_{0}$ as input, one can recover $g$ and $U$ in polynomial time.

Proof. Without loss of generality we can assume $g \leq U$, since otherwise we can apply the algorithm with $U \omega^{-1} \equiv g\left(\bmod x_{0}\right)$. Let $B \in \mathbb{Z}_{\geq 1}$ such that $U \leq B g \leq 2 U$. When the bit size of $g$ and $U$ is unknown, such a $B$ can be found by exhaustive search in polynomial time. We consider the lattice $L \subseteq \mathbb{Z}^{2}$ of vectors $(B x, y)$ such that $x \omega \equiv y\left(\bmod x_{0}\right)$. From $g \omega \equiv U\left(\bmod x_{0}\right)$ it follows that $L$ contains the vector $\boldsymbol{v}=(B g, U)$. We show that $\boldsymbol{v}$ is a shortest non-zero vector in $L$.

By Minkowski's Theorem, we have $\lambda_{1}(L) \leq \sqrt{2 \operatorname{det}(L)}$. From Hadamard's Inequality, with $\operatorname{det}(L)=B x_{0}$, we obtain:

$$
\lambda_{2}(L) \geq \frac{\operatorname{det}(L)}{\lambda_{1}(L)} \geq \frac{\sqrt{\operatorname{det}(L)}}{\sqrt{2}}=\frac{\sqrt{B x_{0}}}{\sqrt{2}}>\sqrt{5 B g U} \geq \sqrt{5} U .
$$

Moreover, we have:

$$
\|\boldsymbol{v}\|=\left((B g)^{2}+U^{2}\right)^{1 / 2} \leq \sqrt{5} U
$$

This implies that $\|\boldsymbol{v}\|<\lambda_{2}(L)$ and therefore $\boldsymbol{v}$ is a multiple of a shortest non-zero vector in $L$ : we write $\boldsymbol{v}=k \boldsymbol{u}$ with $\|\boldsymbol{u}\|=\lambda_{1}(L)$, and $k \in \mathbb{Z} \backslash\{0\}$. Letting $\boldsymbol{u}=\left(B u_{1}, u_{2}\right)$, we have $g=k u_{1}$ and $U=k u_{2}$. Hence $k$ divides both $g$ and $U$. Since $\operatorname{gcd}(g, U)=1$ one has $k= \pm 1$. This shows that $\boldsymbol{v}$ is a shortest non-zero vector of $L$.

By running Lagrange-Gauss reduction on the matrix of row vectors:

$$
\left[\begin{array}{cc}
B & \omega \\
0 & x_{0}
\end{array}\right]
$$

one obtains in polynomial time a length-ordered basis $\left(\boldsymbol{b}_{1}, \boldsymbol{b}_{2}\right)$ of $L$ satisfying $\left\|\boldsymbol{b}_{1}\right\|=\lambda_{1}(L)$ and $\left\|\boldsymbol{b}_{2}\right\|=\lambda_{2}(L)$, which enables to recover $g$ and $U$.

Using the same notations as in Section 2, the integer $g=\prod_{i=1}^{\theta} g_{i}$ has approximate bit size $\theta \cdot \alpha$, while the integer $U$ has an approximate bit size $\gamma-\eta+n_{h}+\rho_{f}+\theta \alpha$. From the condition $g \cdot U<x_{0} / 10$ of Proposition 1, we obtain by dropping the term $\log _{2}(10)$, the simplified condition

$$
\gamma-\eta+n_{h}+\rho_{f}+\theta \cdot \alpha+\theta \cdot \alpha<\gamma .
$$

Writing as previously $\nu=\eta-n_{h}-\rho_{f}$ for the number of bits that can be extracted during zero testing, the attack works under the condition:

$$
2 \alpha \theta<\nu
$$

where $\alpha$ is the bit size of the $g_{i}$ 's. In the next section we describe a high-dimensional lattice reduction attack with an improved bound on $\theta$. 


\section{An extended attack against CLT13 with Independent Slots}

Outline of our new attack. Our new attack improves the bound on $\theta$ compared to the attack recalled in Section 3; it also enables to recover multiples of the underlying plaintext messages, instead of only the CLT13 plaintext ring. The main difference is that we work with several messages instead of a single one, using high-dimensional lattice reduction instead of dimension 2 .

Let $\ell \geq 1$ be an integer. Assume that we have $\ell$ level- $\kappa$ encodings $c_{j}$ of plaintext elements $\boldsymbol{m}_{j}=\left(m_{j 1}, \ldots, m_{j n}\right)$ for $1 \leq j \leq \ell$, where each message has non-zero support of length $\theta$. Without loss of generality, we can assume that $m_{j i}=0$ for all $\theta+1 \leq i \leq n$ and all $1 \leq j \leq \ell$. We consider the zero-testing evaluations $\omega_{j}=p_{z t} \cdot c_{j} \bmod x_{0}$ of these encodings, which gives as previously:

$$
\omega_{j} \equiv \sum_{i=1}^{\theta} h_{i}\left(r_{j i}+m_{j i}\left(g_{i}^{-1} \bmod p_{i}\right)\right) \frac{x_{0}}{p_{i}}+\sum_{i=\theta+1}^{n} h_{i} r_{j i} \frac{x_{0}}{p_{i}} \quad\left(\bmod x_{0}\right), \quad 1 \leq j \leq \ell
$$

for integers $r_{j i}$. We can rewrite the above equation as:

$$
\omega_{j} \equiv \sum_{i=1}^{\theta} \alpha_{i} \cdot m_{j i}+R_{j} \quad\left(\bmod x_{0}\right), \quad 1 \leq j \leq \ell
$$

for some integers $\alpha_{i}$, where for each evaluation $\omega_{j}$, the integer $R_{j}$ is significantly smaller than $x_{0}$.

We can see Equation (8) as an instance of a "noisy" hidden subset sum problem. Namely in [NS99], the authors consider the following hidden subset sum problem. Given a positive integer $M$, and a vector $\boldsymbol{b}=\left(b_{1}, \ldots, b_{\ell}\right) \in \mathbb{Z}^{\ell}$ with entries in $[0, M-1]$, find integers $\alpha_{1}, \ldots, \alpha_{n} \in[0, M-1]$ such that there exist vectors $\boldsymbol{x}_{1}, \ldots, \boldsymbol{x}_{n} \in \mathbb{Z}^{\ell}$ with entries in $\{0,1\}$ satisfying:

$$
\boldsymbol{b} \equiv \alpha_{1} \boldsymbol{x}_{1}+\alpha_{2} \boldsymbol{x}_{2}+\cdots+\alpha_{n} \boldsymbol{x}_{n} \quad(\bmod M)
$$

In our case, the weights $\alpha_{1}, \ldots, \alpha_{n}$ are hidden as in [NS99], but for each equation we have an additional hidden noisy term $R_{j}$. Moreover, the weights $\alpha_{i}=h_{i} \cdot\left(g_{i}^{-1} \bmod p_{i}\right) \cdot x_{0} / p_{i}$ have a special structure, instead of being random in [NS99]. Thanks to this special structure, using a variant of the orthogonal lattice approach from [NS99], we can recover the secret product $g=g_{1} \cdots g_{\theta}$ and the plaintext elements $m_{j i}$ up to a scaling factor.

\subsection{Preliminaries on lattices}

Let $L$ be a lattice in $\mathbb{R}^{d}$ of rank $0<n \leq d$. We recall that Hadamard's Inequality gives the following upper bound on the determinant of $L$, for every basis $\mathcal{B}$ of $L$ :

$$
\operatorname{det}(L) \leq \prod_{\boldsymbol{b} \in \mathcal{B}}\|\boldsymbol{b}\|
$$

Based on Hadamard's Inequality, we prove the following simple lemma.

Lemma 2. Let $1 \leq n \leq d$ be integers and let $L \subseteq \mathbb{Z}^{d}$ be a lattice of rank $n$. Let $\boldsymbol{x}_{1}, \ldots, \boldsymbol{x}_{n-1} \in L$ be linearly independent. Then for every vector $\boldsymbol{y} \in L$ not in the linear span of $\boldsymbol{x}_{1}, \ldots, \boldsymbol{x}_{n-1}$, one has $\|\boldsymbol{y}\| \geq \operatorname{det}(L) / \prod_{i=1}^{n-1}\left\|\boldsymbol{x}_{i}\right\|$.

Proof. Since $\boldsymbol{x}_{1}, \ldots, \boldsymbol{x}_{n-1}, \boldsymbol{y} \in L$ are linearly independent, they generate a rank-n sublattice $L^{\prime}$ of $L$ and hence $\operatorname{det}(L) \leq \operatorname{det}\left(L^{\prime}\right)$ as $\operatorname{det}(L)$ divides $\operatorname{det}\left(L^{\prime}\right)$. By Hadamard's Inequality, $\operatorname{det}(L) \leq$ $\operatorname{det}\left(L^{\prime}\right) \leq\|\boldsymbol{y}\| \cdot \prod_{i=1}^{n-1}\left\|\boldsymbol{x}_{i}\right\|$. The bound follows.

We recall that the LLL algorithm [LLL82], given an input basis of $L$, produces a reduced basis of $L$ with respect to the choice of a parameter $\delta \in(1 / 4,1)$; we call such a basis $\delta$-reduced. More precisely, we will use the following theorem. 
Theorem 3. Let $1 \leq n \leq d$ be integers and let $L \subseteq \mathbb{Z}^{d}$ be a lattice of rank $n$. Let $\left\{\boldsymbol{b}_{i}: 1 \leq i \leq n\right\}$ be a basis of $L$. Let $B \in \mathbb{Z}_{\geq 1}$ be such that $\left\|\boldsymbol{b}_{i}\right\|^{2} \leq B$ for $1 \leq i \leq n$. Let $\delta \in(1 / 4,1)$. Then the LLL algorithm with reduction parameter $\delta$ outputs a $\delta$-reduced basis $\left\{\boldsymbol{b}_{i}^{\prime}: 1 \leq i \leq n\right\}$ after $\mathcal{O}\left(n^{5} d \log ^{3} B\right)$ operations. Moreover, the first vector in such a basis satisfies:

$$
\left\|\boldsymbol{b}_{1}^{\prime}\right\| \leq c^{(n-1) / 2}\|\boldsymbol{x}\|
$$

for every non-zero $\boldsymbol{x} \in L$, and where $c=1 /(\delta-1 / 4)$.

\subsection{Our first lattice-based attack}

Setting. In this section, we describe our first attack based on a variant of the hidden subset-sum problem. We consider plaintext elements $\boldsymbol{m}_{1}, \ldots, \boldsymbol{m}_{\ell} \in \mathbb{Z}^{n}$ and write $m_{j i}$ for the $i$-th entry of the $j$-th message, where $0 \leq m_{j i}<g_{i}$ for all $1 \leq i \leq n$ and $1 \leq j \leq \ell$. As previously, we assume that $m_{j i}=0$ for all $\theta+1 \leq i \leq n$. We write $\boldsymbol{M}$ for the matrix of row vectors $\boldsymbol{m}_{j}$ for $1 \leq j \leq \ell$; and we will denote its columns by $\hat{\boldsymbol{m}}_{i}$ for $1 \leq i \leq n$, that is, $\boldsymbol{M}=\left[\hat{\boldsymbol{m}}_{1}|\cdots| \hat{\boldsymbol{m}}_{n}\right] \in \operatorname{Mat}_{\ell \times n}(\mathbb{Z})$. By construction, the vectors $\hat{\boldsymbol{m}}_{i}$ for $\theta+1 \leq i \leq n$ are all zero. We also assume that for all $1 \leq i \leq \theta$, $\hat{\boldsymbol{m}}_{i} \not \equiv \mathbf{0}\left(\bmod g_{i}\right)$. For $1 \leq j \leq \ell$, we let $c_{j}$ denote an encoding of $\boldsymbol{m}_{j}$ at the last level $\kappa$ :

$$
c_{j} \equiv \frac{r_{j i} g_{i}+m_{j i}}{z^{\kappa}} \quad\left(\bmod p_{i}\right), \quad 1 \leq i \leq n
$$

where $r_{j i} \in \mathbb{Z}$ are $\rho_{f}$-bit integers. Letting $\boldsymbol{c}=\left(c_{j}\right)_{1 \leq j \leq \ell}$, this gives a vector equation over $\mathbb{Z}^{\ell}$ :

$$
\boldsymbol{c} \equiv z^{-\kappa}\left(g_{i} \boldsymbol{r}_{i}+\hat{\boldsymbol{m}}_{i}\right) \quad\left(\bmod p_{i}\right), \quad 1 \leq i \leq n
$$

for $\boldsymbol{r}_{i}=\left(r_{j i}\right)_{1 \leq j \leq \ell}$. Let $p_{z t}$ be the zero-testing parameter, as defined in (3). From zero-testing we obtain the following equations:

$$
\omega_{j} \equiv c_{j} \cdot p_{z t} \equiv \sum_{i=1}^{\theta} h_{i} m_{j i}\left(g_{i}^{-1} \bmod p_{i}\right) \frac{x_{0}}{p_{i}}+\sum_{i=1}^{n} h_{i} r_{j i} \frac{x_{0}}{p_{i}} \quad\left(\bmod x_{0}\right), 1 \leq j \leq \ell
$$

which can be rewritten as $\omega_{j} \equiv \sum_{i=1}^{\theta} \alpha_{i} m_{j i}+R_{j}\left(\bmod x_{0}\right)$, where we use the shorthand notations:

$$
\alpha_{i}:=h_{i}\left(g_{i}^{-1} \bmod p_{i}\right) \frac{x_{0}}{p_{i}}, 1 \leq i \leq \theta
$$

and $R_{j}:=\sum_{i=1}^{n} h_{i} r_{j i} \frac{x_{0}}{p_{i}}$ for $1 \leq j \leq \ell$. As a vector equation, this reads:

$$
\boldsymbol{\omega} \equiv p_{z t} \cdot \boldsymbol{c} \equiv \sum_{i=1}^{\theta} \alpha_{i} \hat{\boldsymbol{m}}_{i}+\boldsymbol{R} \quad\left(\bmod x_{0}\right)
$$

with $\boldsymbol{\omega}=\left(\omega_{j}\right)_{1 \leq j \leq \ell}$; for $1 \leq i \leq \theta$ the vectors $\hat{\boldsymbol{m}}_{i}$ are as above and $\boldsymbol{R}=\left(R_{j}\right)_{1 \leq j \leq \ell}=\sum_{i=1}^{n} h_{i} \frac{x_{0}}{p_{i}} \boldsymbol{r}_{i}$.

In the above equation, the components of $\boldsymbol{R}$ have approximate bit size $\rho_{R}=\gamma-\eta+n_{h}+\rho_{f}$. Using, as previously, $\nu=\eta-n_{h}-\rho_{f}$ as the number of bits that can be extracted, we have therefore $\rho_{R}=\gamma-\nu$. As explained above, Equation (11) is similar to an instance of the hidden subset sum problem, so we describe a variant of the orthogonal lattice attack from [NS99], which recovers the secret CLT13 plaintext ring and the hidden plaintexts $\left\{\hat{\boldsymbol{m}}_{i}: 1 \leq i \leq \theta\right\}$, up to a scaling factor. For the sequel, we assume that the prime numbers $g_{1}, \ldots, g_{\theta}$ are distinct, and that for every $1 \leq i \leq \theta$, we have $\operatorname{gcd}\left(g_{i}, h_{i} x_{0} / p_{i}\right)=1$. 
The orthogonal lattice $\boldsymbol{L}$. We consider the lattice $L$ of vectors $(B \boldsymbol{u}, v) \in \mathbb{Z}^{\ell+1}$, with $\boldsymbol{u} \in \mathbb{Z}^{\ell}$ and $v \in \mathbb{Z}$, such that $(\boldsymbol{u}, v)$ is orthogonal to $(\boldsymbol{\omega}, 1)$ modulo $x_{0}$, where $B \in \mathbb{Z}_{\geq 1}$ is a scaling factor that will be determined later. Since $L$ contains the sublattice $x_{0} \mathbb{Z}^{\ell+1}$, it has full-rank $\ell+1$. We note that this lattice is known (i.e. we can construct a basis for it) since $\boldsymbol{\omega}$ and $x_{0}$ are given. Our attack is based on the fact that $L$ contains a rank- $\ell$ sublattice $L^{\prime}$, generated by reasonably short vectors $\left\{\left(B \boldsymbol{u}_{i}, v_{i}\right): 1 \leq i \leq \ell\right\}$ of $L$, which can be used to reveal the secret product $g=\prod_{i=1}^{\theta} g_{i}$.

More precisely, for every $(B \boldsymbol{u}, v) \in L$, we obtain from (11):

$$
\langle\boldsymbol{u}, \boldsymbol{\omega}\rangle+v \equiv \sum_{i=1}^{\theta} \alpha_{i}\left\langle\boldsymbol{u}, \hat{\boldsymbol{m}}_{i}\right\rangle+\langle\boldsymbol{u}, \boldsymbol{R}\rangle+v \equiv 0 \quad\left(\bmod x_{0}\right)
$$

and therefore, the vector $\left(\left\langle\boldsymbol{u}, \hat{\boldsymbol{m}}_{1}\right\rangle, \ldots,\left\langle\boldsymbol{u}, \hat{\boldsymbol{m}}_{\theta}\right\rangle,\langle\boldsymbol{u}, \boldsymbol{R}\rangle+v\right)$ is orthogonal modulo $x_{0}$ to the vector $\boldsymbol{a}=\left(\alpha_{1}, \ldots, \alpha_{\theta}, 1\right)$. To obtain balanced components, we use another scaling factor $C \in \mathbb{Z}_{\geq 1}$ and we consider the vector:

$$
\boldsymbol{p}_{\boldsymbol{u}, v}:=\left(C\left\langle\boldsymbol{u}, \hat{\boldsymbol{m}}_{1}\right\rangle, \ldots, C\left\langle\boldsymbol{u}, \hat{\boldsymbol{m}}_{\theta}\right\rangle,\langle\boldsymbol{u}, \boldsymbol{R}\rangle+v\right)
$$

Following the original orthogonal lattice attack from [NS99], if a vector $(B \boldsymbol{u}, v) \in L$ is short enough, then the associated vector $\boldsymbol{p}_{\boldsymbol{u}, v}=(C \boldsymbol{x}, y)$ will also be short, and if $(\boldsymbol{x}, y)$ becomes shorter than a shortest non-zero vector orthogonal to $\boldsymbol{a}$ modulo $x_{0}$, we must have $\boldsymbol{p}_{\boldsymbol{u}, v}=0$, which implies $\left\langle\boldsymbol{u}, \hat{\boldsymbol{m}}_{i}\right\rangle=0$ for all $1 \leq i \leq \theta$. We will see that in our setting, because of the specific structure of the coefficients $\alpha_{i}$ 's, we only get $\left\langle\boldsymbol{u}, \hat{\boldsymbol{m}}_{i}\right\rangle \equiv 0\left(\bmod g_{i}\right)$ for all $1 \leq i \leq \theta$. Therefore, by applying lattice reduction to $L$, we expect to recover the lattice $\Lambda^{\perp}$ of vectors $\boldsymbol{u}$ which are orthogonal to all $\hat{\boldsymbol{m}}_{i}$ modulo $g_{i}$; since by assumption $\hat{\boldsymbol{m}}_{i} \not \equiv \mathbf{0}\left(\bmod g_{i}\right)$ for all $1 \leq i \leq \theta$, the lattice $\Lambda_{i}^{\perp}=\left\{\boldsymbol{u} \in \mathbb{Z}^{\ell}:\left\langle\boldsymbol{u}, \hat{\boldsymbol{m}}_{i}\right\rangle \equiv 0\right.$ $\left.\left(\bmod g_{i}\right)\right\}$ has determinant $g_{i}$, and since $g_{1}, \ldots, g_{\theta}$ are distinct primes, the lattice $\Lambda^{\perp}=\cap_{i=1}^{\theta} \Lambda_{i}^{\perp}$ has determinant equal to $g=\prod_{i=1}^{\theta} g_{i}$. In particular, any basis for this lattice reveals $g$ by computing its determinant.

The lattice $\boldsymbol{A}^{\perp}$. Henceforth, we must study the short vectors in the lattice of vectors orthogonal to $\boldsymbol{a}$ modulo $x_{0}$. More precisely, we consider the lattice $A^{\perp}$ of vectors $(C \boldsymbol{x}, y) \in \mathbb{Z}^{\theta+1}$, such that $(\boldsymbol{x}, y)$ is orthogonal to $\boldsymbol{a}=\left(\alpha_{1}, \ldots, \alpha_{\theta}, 1\right)$ modulo $x_{0}$; therefore $\boldsymbol{p}_{\boldsymbol{u}, v} \in A^{\perp}$. The lattice $A^{\perp}$ has full-rank $\theta+1$ and we have $\operatorname{det}\left(A^{\perp}\right)=C^{\theta} x_{0}$. Namely, we have an abstract group isomorphism $A^{\perp} \simeq(C \mathbb{Z})^{\theta} \oplus x_{0} \mathbb{Z}$, sending $(C \boldsymbol{x}, y)$ to $(C \boldsymbol{x},\langle\boldsymbol{x}, \boldsymbol{a}\rangle+y)$.

As mentioned previously, the coefficients $\alpha_{i}$ 's in the vector $\boldsymbol{a}$ have a particular structure. Namely, we have $\alpha_{i}=\left(g_{i}^{-1} \bmod p_{i}\right) h_{i} x_{0} / p_{i}$, and therefore

$$
g_{i} \cdot \alpha_{i} \equiv h_{i} \cdot \frac{x_{0}}{p_{i}} \quad\left(\bmod x_{0}\right)
$$

for all $1 \leq i \leq \theta$. Therefore the lattice $A^{\perp}$ contains the $\theta$ linearly independent short vectors $\boldsymbol{q}_{i}=$ $\left(0, \ldots, 0, C g_{i}, 0, \ldots, 0,-s_{i}\right)$, where $s_{i}=h_{i} \cdot x_{0} / p_{i}$. Using $C:=2^{\rho_{R}-\alpha}$, we get $\left\|\boldsymbol{q}_{i}\right\| \simeq C \cdot 2^{\alpha}$.

We now derive a condition on $\left\|\boldsymbol{p}_{\boldsymbol{u}, v}\right\|$ so that the vector $\boldsymbol{p}_{\boldsymbol{u}, v}$ belongs to the sublattice of $A^{\perp}$ generated by the short vectors $\left\{\boldsymbol{q}_{i}: 1 \leq i \leq \theta\right\}$. From Lemma 2 , if $\left\|\boldsymbol{p}_{\boldsymbol{u}, v}\right\|<\operatorname{det}\left(A^{\perp}\right) / \prod_{i=1}^{\theta}\left\|\boldsymbol{q}_{i}\right\|$, then $\boldsymbol{p}_{\boldsymbol{u}, v}$ must belong to the linear span generated by the $\boldsymbol{q}_{i}$ 's; since by assumption, the $g_{i}$ 's are distinct primes and $\operatorname{gcd}\left(s_{i}, g_{i}\right)=1$ for all $1 \leq i \leq \theta$, this implies that it must belong to the sublattice generated by the $\boldsymbol{q}_{i}$ 's. In that case, we have:

$$
\left\langle\boldsymbol{u}, \hat{\boldsymbol{m}}_{i}\right\rangle \equiv 0 \quad\left(\bmod g_{i}\right), \quad 1 \leq i \leq \theta
$$

From $\operatorname{det}\left(A^{\perp}\right)=C^{\theta} \cdot x_{0}$ and $\left\|\boldsymbol{q}_{i}\right\| \simeq C \cdot 2^{\alpha}$, the previous condition $\left\|\boldsymbol{p}_{\boldsymbol{u}, v}\right\|<\operatorname{det}\left(A^{\perp}\right) / \prod_{i=1}^{\theta}\left\|\boldsymbol{q}_{i}\right\|$ gives the approximate condition:

$$
\left\|\boldsymbol{p}_{\boldsymbol{u}, v}\right\|<2^{\gamma-\alpha \cdot \theta}
$$


Short vectors in $\boldsymbol{L}$. We now study the short vectors of $L$; more precisely, we explain that $L$ contains $\ell$ linearly independent short vectors of norm roughly $2^{\rho_{R}+\alpha \theta / \ell}$. We show that these vectors can be derived from the lattice $\Lambda^{\perp}$ of vectors $\boldsymbol{u} \in \mathbb{Z}^{\ell}$ satisfying (12), i.e. that are orthogonal to $\hat{\boldsymbol{m}}_{i}$ modulo $g_{i}$ for every $1 \leq i \leq \theta$. This is a full-rank lattice of dimension $\ell$ and determinant $g=\prod_{i=1}^{\theta} g_{i}$, with $g \simeq 2^{\alpha \theta}$. Therefore, we heuristically expect that the lattice $\Lambda^{\perp}$ contains $\ell$ linearly independent vectors of norm roughly $\left(\operatorname{det} \Lambda^{\perp}\right)^{1 / \ell} \simeq 2^{\alpha \theta / \ell}$. We show that from any short $\boldsymbol{u} \in \Lambda^{\perp}$, we can generate a vector $(\boldsymbol{u}, v)$ with small $v$, and orthogonal to $(\boldsymbol{\omega}, 1)$ modulo $x_{0}$, and consequently a short vector $(B \boldsymbol{u}, v) \in L$. For this, we write $\left\langle\boldsymbol{u}, \hat{\boldsymbol{m}}_{i}\right\rangle=k_{i} g_{i}$ with $k_{i} \in \mathbb{Z}$, and we have:

$$
\begin{aligned}
\langle\boldsymbol{u}, \boldsymbol{\omega}\rangle+v & \equiv \sum_{i=1}^{\theta} \alpha_{i}\left\langle\boldsymbol{u}, \hat{\boldsymbol{m}}_{i}\right\rangle+\langle\boldsymbol{u}, \boldsymbol{R}\rangle+v \equiv \sum_{i=1}^{\theta} k_{i} \cdot g_{i} \cdot \alpha_{i}+\langle\boldsymbol{u}, \boldsymbol{R}\rangle+v \quad\left(\bmod x_{0}\right) \\
& \equiv \sum_{i=1}^{\theta} k_{i} \cdot s_{i}+\langle\boldsymbol{u}, \boldsymbol{R}\rangle+v \quad\left(\bmod x_{0}\right)
\end{aligned}
$$

Therefore, it suffices to let $v:=-\langle\boldsymbol{u}, \boldsymbol{R}\rangle-\sum_{i=1}^{\theta} k_{i} \cdot s_{i}$ to obtain $\langle\boldsymbol{u}, \boldsymbol{\omega}\rangle+v \equiv 0\left(\bmod x_{0}\right)$; the vector $(\boldsymbol{u}, v)$ is then orthogonal to $(\boldsymbol{\omega}, 1)$ modulo $x_{0}$, and thus $(B \boldsymbol{u}, v) \in L$. We obtain $|v| \simeq\|\boldsymbol{u}\| \cdot 2^{\rho_{R}}$; therefore letting $B:=2^{\rho_{R}}$, we get $\|(B \boldsymbol{u}, v)\| \simeq 2^{\rho_{R}}\|\boldsymbol{u}\|$. In summary, the lattice $L$ contains a sublattice $L^{\prime}$ of rank $\ell$, generated by $\ell$ vectors of norm roughly $2^{\rho_{R}+\alpha \theta / \ell}$. That the recovered vectors are indeed linearly independent is the content of the following lemma, which we prove in Appendix A.1.

Lemma 4. Let $\left\{\left(B \boldsymbol{u}_{j}, v_{j}\right): 1 \leq j \leq \ell+1\right\}$ be a basis of the lattice $L$ and assume that the vectors $\left\{\boldsymbol{p}_{\boldsymbol{u}_{j}, v_{j}}: 1 \leq j \leq \ell\right\}$ belong to the sublattice of $A^{\perp}$ generated by the vectors $\left\{\boldsymbol{q}_{i}: 1 \leq i \leq \theta\right\}$. Then the vectors $\left\{\boldsymbol{u}_{j}: 1 \leq j \leq \ell\right\}$ are $\mathbb{R}$-linearly independent.

Recovering $\boldsymbol{g}=\prod_{i=1}^{\theta} \boldsymbol{g}_{\boldsymbol{i}}$. By applying lattice reduction to the lattice $L$, we expect that the first $\ell$ vectors $\left\{\left(B \boldsymbol{u}_{j}, v_{j}\right): 1 \leq j \leq \ell\right\}$ of a reduced basis belong to the above sublattice $L^{\prime}$ and have norm roughly:

$$
\left\|\left(B \boldsymbol{u}_{j}, v_{j}\right)\right\| \simeq 2^{\rho_{R}+\alpha \theta / \ell} \cdot 2^{\iota(\ell+1)}, 1 \leq j \leq \ell
$$

where $2^{\iota(\ell+1)}$ is the Hermite factor for some positive constant $\iota$ depending on the lattice reduction algorithm. With $C=2^{\rho_{R}-\alpha}$, we have $\left\|\boldsymbol{p}_{\boldsymbol{u}_{i}, v_{i}}\right\| \simeq\left\|\left(B \boldsymbol{u}_{i}, v_{i}\right)\right\|$ for all $1 \leq i \leq \ell$. From the condition given by (13), we have that $\boldsymbol{u}_{i} \in \Lambda^{\perp}$ if $\left\|\boldsymbol{p}_{\boldsymbol{u}_{i}, v_{i}}\right\|<2^{\gamma-\alpha \cdot \theta}$; therefore combining with (14) we get the approximate condition:

$$
\rho_{R}+\frac{\alpha \theta}{\ell}+\iota(\ell+1)<\gamma-\alpha \theta
$$

Using $\rho_{R}=\gamma-\nu$ where $\nu$ is the number of bits that can be extracted from zero-testing, this condition becomes

$$
\alpha \theta\left(1+\frac{1}{\ell}\right)+\iota(\ell+1)<\nu
$$

In summary, when Condition (15) is satisfied, we expect to recover a basis $\left\{\boldsymbol{u}_{i}: 1 \leq i \leq \ell\right\}$ of the lattice $\Lambda^{\perp}$; then since $\operatorname{det}\left(\Lambda^{\perp}\right)=g=\prod_{i=1}^{\theta} g_{i}$, the absolute value of the determinant of the basis matrix reveals $g$.

From Equation (15), we observe that the parameter $\ell$ can be kept relatively small (say $\ell \simeq 10$ ), as larger values of $\ell$ would not significantly improve the bound; this implies that the lattice dimension $\ell+1$ on which LLL is applied can be kept relatively small. Moreover for LLL, experiments show that $2^{\iota} \simeq 1.021$ so that $\iota$ is approximately 0.03 , and therefore for such small values of $\ell$, the term $\iota \cdot(\ell+1)$ is negligible. Thus we can use the simpler approximate bound for our attack:

$$
\alpha \theta<\nu
$$


This gives a factor 2 improvement compared to the previous bound given by (7), following the attack of [GLW14]. In the next subsection we will see how to get a much more significant improvement, with $\alpha \theta=\mathcal{O}\left(\nu^{2}\right)$.

A proven variant. The above algorithm is heuristic only. Below we describe a proven variant that can recover a vector $\boldsymbol{u}$ such that $\left\langle\boldsymbol{u}, \hat{\boldsymbol{m}}_{i}\right\rangle \equiv 0\left(\bmod g_{i}\right)$ for all $1 \leq i \leq \theta$, using the LLL reduction algorithm. Although we only recover a single vector $\boldsymbol{u}$ instead of a lattice basis, this will be enough when combined with the Cheon et al. attack to recover all secret parameters of CLT13 (see Section 5). We provide the proof of Proposition 5 in Appendix A.2.

Proposition 5. Let $\ell, \theta \in \mathbb{Z}_{\geq 1}, x_{0} \in \mathbb{Z}_{\geq 1}$ and let $g_{i} \in \mathbb{Z}_{\geq 2}$ be distinct $\alpha$-bit prime numbers for $1 \leq i \leq \theta$ and some $\alpha \in \mathbb{Z}_{\geq 1}$. For $1 \leq i \leq \theta$, let $\alpha_{i} \in \mathbb{Z}$ such that $g_{i} \cdot \alpha_{i} \equiv s_{i}\left(\bmod x_{0}\right)$, for $s_{i} \in \mathbb{Z}$ satisfying $\left|s_{i}\right| \leq 2^{\rho_{R}}$, for some $\rho_{R} \in \mathbb{Z}_{\geq 1}$ and assume that $\operatorname{gcd}\left(g_{i}, s_{i}\right)=1$. For $1 \leq i \leq \theta$, let $\hat{\boldsymbol{m}}_{i} \in \mathbb{Z}^{\ell}$ be vectors with entries in $\left[0, g_{i}\right) \cap \mathbb{Z}$ such that $\hat{\boldsymbol{m}}_{i} \not \equiv \mathbf{0}\left(\bmod g_{i}\right)$, and let $\boldsymbol{R} \in \mathbb{Z}^{\ell}$ such that $\|\boldsymbol{R}\|_{\infty} \leq 2^{\rho_{R}}$. Let $\boldsymbol{\omega} \in \mathbb{Z}^{\ell}$ such that $\boldsymbol{\omega} \equiv \sum_{i=1}^{\theta} \alpha_{i} \hat{\boldsymbol{m}}_{i}+\boldsymbol{R}\left(\bmod x_{0}\right)$. Assume that

$$
\alpha \theta\left(1+\frac{1}{\ell}\right)+\frac{\ell+\theta}{2}+\log _{2}(\ell \sqrt{\ell+1} \cdot \theta)+4<\log _{2}\left(x_{0}\right)-\rho_{R} .
$$

Given the integers $\ell, \theta, \rho_{R}, x_{0}$ and the vector $\boldsymbol{\omega}$, one can recover in polynomial time a vector $\boldsymbol{u} \in \mathbb{Z}^{\ell}$ such that $\left\langle\boldsymbol{u}, \hat{\boldsymbol{m}}_{i}\right\rangle \equiv 0\left(\bmod g_{i}\right)$ for all $1 \leq i \leq \theta$, satisfying $\|\boldsymbol{u}\| \leq 2^{\ell / 2} \sqrt{\ell(\ell+1)}\left(\prod_{i=1}^{\theta} g_{i}\right)^{1 / \ell}$.

We remark that by replacing $\log _{2}\left(x_{0}\right)-\rho_{R}$ by $\gamma-\rho_{R}=\nu$, we recover, up to additional logarithmic terms, the approximate bound established in (15).

\subsection{Extended Orthogonal Lattice Attack}

In this section we describe an extended attack that significantly improves the bound on $\theta$ established in (16). Let $\ell, d \geq 1$ be integers. As previously, we assume that we have encodings $c_{j}$ of plaintext elements $\boldsymbol{m}_{j}=\left(m_{j 1}, \ldots, m_{j n}\right)$ for $1 \leq j \leq \ell$, where only the first $\theta$ components of each $\boldsymbol{m}_{j}$ are non-zero, that is, $m_{j i}=0$ for $\theta+1 \leq i \leq n$. However, we assume that these encodings are at level $\kappa-1$, and that we also have an additional set of $d$ level-1 encodings $\left\{c_{k}^{\prime}: 1 \leq k \leq d\right\}$ of plaintext elements $\boldsymbol{x}_{k}=\left(x_{k 1}, \ldots, x_{k n}\right)$ for $1 \leq k \leq d$. By computing the top-level $\kappa$ product encodings, we can therefore obtain the following zero-testing evaluations:

$$
\omega_{j k} \equiv\left(c_{j} \cdot c_{k}^{\prime}\right) \cdot p_{z t} \equiv \sum_{i=1}^{\theta} h_{i} m_{j i} x_{k i}\left(g_{i}^{-1} \bmod p_{i}\right) \frac{x_{0}}{p_{i}}+\sum_{i=1}^{n} h_{i} r_{j k i} \frac{x_{0}}{p_{i}} \quad\left(\bmod x_{0}\right)
$$

for some integers $r_{j k i}$. Since every encoding $c_{j}$ encodes a message with non-zero support of length $\theta$, the product encodings $c_{j} c_{k}^{\prime}$ maintain their zero slots. Note that the same remains valid if the encodings $c_{j}$ are at even lower levels, because they can be raised to level $\kappa-1$ without removing their zero slots. As previously, we rewrite Equation (18) as:

$$
\omega_{j k} \equiv \sum_{i=1}^{\theta} \alpha_{i k} m_{j i}+R_{j k} \quad\left(\bmod x_{0}\right)
$$

where we let

$$
\alpha_{i k}=h_{i} x_{k i}\left(g_{i}^{-1} \bmod p_{i}\right) \frac{x_{0}}{p_{i}}, \quad 1 \leq i \leq \theta, 1 \leq k \leq d
$$

and $R_{j k}=\sum_{i=1}^{n} h_{i} r_{j k i} x_{0} / p_{i}$ for all $1 \leq j \leq \ell$ and $1 \leq k \leq d$. As before, for $1 \leq i \leq \theta$, we denote by $\hat{\boldsymbol{m}}_{i} \in \mathbb{Z}^{\ell}$ the vector with components $m_{j i}$ for $1 \leq j \leq \ell$, and similarly $\boldsymbol{\omega}_{k}$ and $\boldsymbol{R}_{k}$ the 
corresponding vectors in $\mathbb{Z}^{\ell}$. We assume that $\hat{\boldsymbol{m}}_{i} \not \equiv \mathbf{0}\left(\bmod g_{i}\right)$ for all $i$. The previous equation can then be rewritten as:

$$
\boldsymbol{\omega}_{k} \equiv \sum_{i=1}^{\theta} \alpha_{i k} \hat{\boldsymbol{m}}_{i}+\boldsymbol{R}_{k} \quad\left(\bmod x_{0}\right)
$$

The difference with Equation (11) from our first lattice attack is that the vectors $\left\{\hat{\boldsymbol{m}}_{i}: 1 \leq i \leq \theta\right\}$ now satisfy $d$ equations for $1 \leq k \leq d$, instead of a single equation, as in Subsection 4.2. With more constraints on the vectors $\hat{\boldsymbol{m}}_{i}$, we can therefore break the countermeasure from [GLW14] for much higher values of $\theta$. In order to derive a condition on the parameters, we proceed as previously. Namely, the lattices that we considered in Subsection 4.2 now admit natural higher-dimensional analogues.

The orthogonal lattice $\boldsymbol{L}$. As previously, for a scaling factor $B \in \mathbb{Z}_{\geq 1}$, we consider the lattice $L$ of vectors $(B \boldsymbol{u}, \boldsymbol{v}) \in \mathbb{Z}^{\ell+d}$, with $\boldsymbol{u} \in \mathbb{Z}^{\ell}$ and $\boldsymbol{v} \in \mathbb{Z}^{d}$, such that $(\boldsymbol{u}, \boldsymbol{v})$ is orthogonal to the $d$ vectors $\left\{\left(\boldsymbol{\omega}_{k}, \boldsymbol{e}_{k}\right): 1 \leq k \leq d\right\}$ modulo $x_{0}$, where $\boldsymbol{e}_{k} \in \mathbb{Z}^{d}$ is the $k$ th unit vector for $1 \leq k \leq d$. This gives for all $1 \leq k \leq d$ and all $(B \boldsymbol{u}, \boldsymbol{v}) \in L$, writing $\boldsymbol{v}=\left(v_{1}, \ldots, v_{d}\right)$ :

$$
\left\langle\boldsymbol{u}, \boldsymbol{\omega}_{k}\right\rangle+v_{k} \equiv \sum_{i=1}^{\theta} \alpha_{i k}\left\langle\boldsymbol{u}, \hat{\boldsymbol{m}}_{i}\right\rangle+\left\langle\boldsymbol{u}, \boldsymbol{R}_{k}\right\rangle+v_{k} \equiv 0 \quad\left(\bmod x_{0}\right)
$$

and therefore the vector $\left(\left\langle\boldsymbol{u}, \hat{\boldsymbol{m}}_{1}\right\rangle, \ldots,\left\langle\boldsymbol{u}, \hat{\boldsymbol{m}}_{\theta}\right\rangle,\left\langle\boldsymbol{u}, \boldsymbol{R}_{1}\right\rangle+v_{1}, \ldots,\left\langle\boldsymbol{u}, \boldsymbol{R}_{d}\right\rangle+v_{d}\right)$ is orthogonal modulo $x_{0}$ to the $d$ vectors $\boldsymbol{a}_{k}=\left(\alpha_{1 k}, \ldots, \alpha_{\theta k}, \boldsymbol{e}_{k}\right)$, for $1 \leq k \leq d$. Again, using a scaling factor $C \in \mathbb{Z}_{\geq 1}$, we let

$$
\boldsymbol{p}_{\boldsymbol{u}, \boldsymbol{v}}=\left(C\left\langle\boldsymbol{u}, \hat{\boldsymbol{m}}_{1}\right\rangle, \ldots, C\left\langle\boldsymbol{u}, \hat{\boldsymbol{m}}_{\theta}\right\rangle,\left\langle\boldsymbol{u}, \boldsymbol{R}_{1}\right\rangle+v_{1}, \ldots,\left\langle\boldsymbol{u}, \boldsymbol{R}_{d}\right\rangle+v_{d}\right) .
$$

The lattice $\boldsymbol{A}^{\perp}$. In order to bound the norm of the vector $\boldsymbol{p}_{\boldsymbol{u}, \boldsymbol{v}}$, we must study the short vectors in the lattice of vectors orthogonal to the vectors $\boldsymbol{a}_{k}$ modulo $x_{0}$ (instead of single vector $\boldsymbol{a}$ ). As previously, we consider the lattice $A^{\perp}$ of vectors $(C \boldsymbol{x}, \boldsymbol{y}) \in \mathbb{Z}^{\theta+d}$ such that $(\boldsymbol{x}, \boldsymbol{y})$ is orthogonal to the $d$ vectors $\left\{\boldsymbol{a}_{k}: 1 \leq k \leq d\right\}$ modulo $x_{0}$; therefore $\boldsymbol{p}_{\boldsymbol{u}, \boldsymbol{v}} \in A^{\perp}$. The lattice $A^{\perp}$ has full-rank $\theta+d$ and determinant $C^{\theta} x_{0}^{d}$. As previously, the coefficients $\alpha_{i k}$ in the vectors $\boldsymbol{a}_{k}$ have a special structure, since they satisfy the congruence relations

$$
g_{i} \cdot \alpha_{i k} \equiv h_{i} \cdot x_{i k} \cdot \frac{x_{0}}{p_{i}} \quad\left(\bmod x_{0}\right)
$$

for all $1 \leq i \leq \theta$ and $1 \leq k \leq d$. Therefore letting $s_{i k}=h_{i} \cdot x_{i k} \cdot x_{0} / p_{i}$, the lattice $A^{\perp}$ contains the $\theta$ short vectors $\boldsymbol{q}_{i}=\left(0, \ldots, 0, C g_{i}, 0, \ldots, 0,-s_{i 1}, \ldots,-s_{i d}\right)$ for $1 \leq i \leq \theta$. Using $C=2^{\rho_{R}-\alpha}$, we get as previously $\left\|\boldsymbol{q}_{i}\right\| \simeq C \cdot 2^{\alpha}$.

We now derive a bound on $\left\|\boldsymbol{p}_{\boldsymbol{u}, \boldsymbol{v}}\right\|$ so that $\boldsymbol{p}_{\boldsymbol{u}, \boldsymbol{v}}$ belongs to the sublattice generated by the $\theta$ vectors $\left\{\boldsymbol{q}_{i}: 1 \leq i \leq \theta\right\}$. We expect a reduced basis of $A^{\perp}$ to have the first $\theta$ vectors with approximately the same norm as the vectors $\left\{\boldsymbol{q}_{i}: 1 \leq i \leq \theta\right\}$, and to have the last $d$ vectors with norm $U$ satisfying $\left(C \cdot 2^{\alpha}\right)^{\theta} \cdot U^{d} \simeq \operatorname{det}\left(A^{\perp}\right)$. Using $\operatorname{det}\left(A^{\perp}\right)=C^{\theta} x_{0}^{d}$, this gives $U \simeq x_{0} / 2^{\alpha \theta / d}$. This implies that, heuristically, if $\left\|\boldsymbol{p}_{\boldsymbol{u}, \boldsymbol{v}}\right\|<U$, then $\boldsymbol{p}_{\boldsymbol{u}, \boldsymbol{v}}$ must belong to the sublattice generated by the $\theta$ vectors $\left\{\boldsymbol{q}_{i}: 1 \leq i \leq \theta\right\}$. As previously, in that case we have that for all $1 \leq i \leq \theta$ :

$$
\left\langle\boldsymbol{u}, \hat{\boldsymbol{m}}_{i}\right\rangle \equiv 0 \quad\left(\bmod g_{i}\right) .
$$

Short vectors in $\boldsymbol{L}$. We now study the short vectors of $L$; as previously, we show that $L$ contains $\ell$ linearly independent short vectors of norm roughly $2^{\rho_{R}+\alpha \theta / \ell}$, which can be derived from the the lattice $\Lambda^{\perp}$ of vectors $\boldsymbol{u} \in \mathbb{Z}^{\ell}$ satisfying (20). Since, as previously, $\Lambda^{\perp}$ heuristically contains $\ell$ linearly 
independent vectors of norm roughly $\left(\operatorname{det} \Lambda^{\perp}\right)^{1 / \ell} \simeq 2^{\alpha \theta / \ell}$, the lattice $L$ contains $\ell$ linearly independent vectors of norm roughly $2^{\rho_{R}+\alpha \theta / \ell}$. Therefore, by applying lattice reduction to the lattice $L$, we expect that the first $\ell$ vectors $\left\{\left(B \boldsymbol{u}_{i}, \boldsymbol{v}_{i}\right): 1 \leq i \leq \ell\right\}$ of the basis have norm roughly:

$$
\left\|\left(B \boldsymbol{u}_{i}, \boldsymbol{v}_{i}\right)\right\| \simeq B \cdot 2^{\alpha \theta / \ell} \cdot 2^{\iota(\ell+d)}
$$

where $2^{\iota(\ell+d)}$ is the Hermite factor. With $B=2^{\rho_{R}}$ and $C=2^{\rho_{R}-\alpha}$, we have $\left\|\boldsymbol{p}_{\boldsymbol{u}_{i}, \boldsymbol{v}_{i}}\right\| \simeq\left\|\left(B \boldsymbol{u}_{\boldsymbol{i}}, \boldsymbol{v}_{i}\right)\right\|$. From the condition $\left\|\boldsymbol{p}_{\boldsymbol{u}_{i}, \boldsymbol{v}_{i}}\right\|<U$, we get the condition:

$$
\rho_{R}+\frac{\alpha \theta}{\ell}+\iota(\ell+d)<\gamma-\frac{\alpha \theta}{d}
$$

which gives using $\rho_{R}=\gamma-\nu$ :

$$
\alpha \theta \cdot\left(\frac{1}{\ell}+\frac{1}{d}\right)+\iota(\ell+d)<\nu
$$

Remark that with $d=1$ the previous bound gives Equation (15). Since (21) is concave and symmetric in both $\ell$ and $d$, the optimum is to take $\ell=d$. This gives the bound:

$$
\frac{2 \alpha \theta}{\ell}+2 \iota \ell<\nu
$$

Recovering $\boldsymbol{g}=\prod_{i=1}^{\boldsymbol{\theta}} \boldsymbol{g}_{\boldsymbol{i}}$. When the above condition is satisfied, as previously we expect to recover a basis $\left\{\boldsymbol{u}_{i}: 1 \leq i \leq \ell\right\}$ of the lattice $\Lambda^{\perp}$. Then since $\operatorname{det}\left(\Lambda^{\perp}\right)=g=\prod_{i=1}^{\theta} g_{i}$, the absolute value of the determinant of the basis matrix reveals $g$. In particular, it follows that the attack requires $\ell>2 \alpha \theta / \nu$, and we must have:

$$
\iota<\frac{\nu^{2}}{4 \alpha \theta}
$$

Heuristically, achieving a Hermite factor of $2^{\iota 2 \ell}$ requires $2^{\Omega(1 / \iota)}$ using BKZ reduction with blocksize $\beta=\omega(1 / \iota)$, [HPS11]. The attack has therefore complexity $2^{\Omega\left(\alpha \theta / \nu^{2}\right)}$; the attack has therefore (heuristic) polynomial-time complexity under the condition:

$$
\alpha \theta=\mathcal{O}\left(\nu^{2}\right)
$$

which significantly improves our previous bound given by (16). Conversely, one expects that the attack is prevented under the condition:

$$
\theta=\omega\left(\frac{\nu^{2}}{\alpha} \log \lambda\right)
$$

In Section 4.5 we provide concrete parameters for CLT13 multilinear maps with independent slots. We will see that Condition (23) requires a much higher value for $\theta$ than the condition $2 \theta \alpha \geq \nu$ for preventing the [GLW14] attack. Namely for $\lambda=80$ bits of security, the bound $2 \theta \alpha \geq \nu$ already holds for $\theta=2$, while a concrete application of Condition (23) requires $\theta \geq 1789$.

Analogy of the attacks. We remark that our extended attacks share similarities with the 2dimensional attack from Section 3. For $\ell, d \in \mathbb{Z}_{\geq 1}$, our extended lattice attack works by reducing the $(\ell+d)$-dimensional lattice

$$
L_{(\ell, d)}=\left\{(B \boldsymbol{u}, \boldsymbol{v}) \in \mathbb{Z}^{\ell} \times \mathbb{Z}^{d}:\left\langle(\boldsymbol{u}, \boldsymbol{v}),\left(\boldsymbol{\omega}_{k}, \boldsymbol{e}_{k}\right)\right\rangle \equiv 0 \quad\left(\bmod x_{0}\right), 1 \leq k \leq d\right\},
$$

where $B \in \mathbb{Z}_{\geq 1}$ is fixed. With this notation, the three attacks work by reducing the lattices $L_{(1,1)}$, $L_{(\ell, 1)}$ and $L_{(\ell, d)}$, respectively. Note that $L_{(1,1)}$ is the lattice $\left\{(B u, v) \in \mathbb{Z}^{2}: u \omega+v \equiv 0\left(\bmod x_{0}\right)\right\}$. For the extended attacks, the $\ell \times \ell$ top-left submatrix of a reduced basis of $L_{(\ell, d)}$ (divided by $B$ ) has determinant $\pm g$. Note that this coincides with the 2-dimensional case $\ell=d=1$ : the first entry (divided by $B$ ) of the first vector in a reduced basis equals $\pm g$ (i.e. a " $1 \times 1$ submatrix" of determinant $\pm g$ ). As such, our higher-dimensional attacks are consistent generalizations of the 2-dimensional attack. 
Summary. We have described a lattice-based attack, which under the condition $\alpha \theta=\mathcal{O}\left(\nu^{2}\right)$, and given as input a collection of encodings (or products of encodings) of messages with non-zero support of length $\theta$, outputs the secret plaintext ring of CLT13. More precisely, our extended lattice attack with the improved bound $\alpha \theta=\mathcal{O}\left(\nu^{2}\right)$ can be described in the following three steps, with parameters $\ell, d \geq 1$. We provide in https://pastebin.com/7WEMHBE9 the source code in Sage [S $\left.\mathrm{S}^{+} 17\right]$.

Input: Sets of level- $\kappa$ encodings $\left\{c_{j} \cdot c_{k}^{\prime} \bmod x_{0}: 1 \leq j \leq \ell, 1 \leq k \leq d\right\}$ where $c_{j}$ encodes a message of non-zero support of length $\theta$.

Output: $g=\prod_{i=1}^{\theta} g_{i}$

1. For $1 \leq k \leq d$, compute the vectors $\boldsymbol{\omega}_{k} \in \mathbb{Z}^{\ell}$ with $\left(\boldsymbol{\omega}_{k}\right)_{j}=c_{j} \cdot c_{k}^{\prime} \cdot p_{z t} \bmod x_{0}$.

2. Let $B=2^{\rho_{R}}$ and compute a LLL-reduced basis of the lattice $L_{(\ell, d)} \subseteq \mathbb{Z}^{\ell+d}$ of vectors $\left\{(B \boldsymbol{u}, \boldsymbol{v}) \in \mathbb{Z}^{\ell} \times \mathbb{Z}^{d}:\left\langle(\boldsymbol{u}, \boldsymbol{v}),\left(\boldsymbol{\omega}_{k}, \boldsymbol{e}_{k}\right)\right\rangle \equiv 0\left(\bmod x_{0}\right), 1 \leq k \leq d\right\}$, where $\boldsymbol{e}_{k} \in \mathbb{Z}^{d}$ is the $k$ th unit vector for $1 \leq k \leq d$. Denote by $\left\{\left(B \boldsymbol{u}_{j}, \boldsymbol{v}_{j}\right): 1 \leq j \leq \ell+d\right\}$ the LLL-reduced basis.

3. Form the $\ell \times \ell$ matrix $\boldsymbol{P}$ of vectors $\left\{\boldsymbol{u}_{j}: 1 \leq j \leq \ell\right\}$ and compute $|\operatorname{det}(\boldsymbol{P})|=g=\prod_{i=1}^{\theta} g_{i}$.

Variant with multiple $\boldsymbol{p}_{\boldsymbol{z t}}$. In many concrete constructions based on composite order multilinear maps, intermediate-level encodings of almost zero plaintexts are not necessarily available. We refer to Section 6 for the application of our attacks to concrete constructions. In order to get around this assumption, we consider a variant of the above attack, where we have multiple zero-testing elements $p_{z t}$ instead of a single one. Namely, as described in [CLT13], in order to get a proper zero-testing procedure, one needs to use a vector of $n$ elements $p_{z t}$. We denote by $p_{z t, k}$ for $1 \leq k \leq n$ those zero-testing elements:

$$
p_{z t, k}=\sum_{i=1}^{n} h_{i k} z^{\kappa}\left(g_{i}^{-1} \bmod p_{i}\right) \frac{x_{0}}{p_{i}} \bmod x_{0}
$$

for corresponding integers $h_{i k}$. As previously, we assume that we have encodings $c_{j}$ of plaintext elements $\boldsymbol{m}_{j}=\left(m_{j 1}, \ldots, m_{j n}\right)$ for $1 \leq j \leq \ell$, where only the first $\theta$ components of each $\boldsymbol{m}_{j}$ are non-zero, that is, $m_{j i}=0$ for $\theta+1 \leq i \leq n$. We can now assume that these encodings are at the last level $\kappa$. Thanks to the multiple zero-testing elements, we can therefore obtain the following zero-testing evaluations:

$$
\omega_{j k} \equiv c_{j} \cdot p_{z t, k} \equiv \sum_{i=1}^{\theta} h_{i k} m_{j i}\left(g_{i}^{-1} \bmod p_{i}\right) \frac{x_{0}}{p_{i}}+\sum_{i=1}^{n} h_{i k} r_{j k i} \frac{x_{0}}{p_{i}} \quad\left(\bmod x_{0}\right)
$$

for some integers $r_{j k i}$, which is similar to (18) with $h_{i k}=h_{i} \cdot x_{k i}$. Therefore the same attack applies and the secret $g=\prod_{i=1}^{\theta} g_{i}$ can be recovered in (heuristic) polynomial-time under the condition $\alpha \theta=\mathcal{O}\left(\nu^{2}\right)$.

\subsection{Revealing information about the plaintext elements}

We show that our attack not only reveals the secret CLT13 plaintext ring, but also information about the secret plaintext elements $\left\{\hat{\boldsymbol{m}}_{i}: 1 \leq i \leq \theta\right\}$. Namely, the orthogonal lattice attack not only recovers $g=\prod_{i=1}^{\theta} g_{i}$, but also constructs a matrix $\boldsymbol{U}$ of rows $\left\{\boldsymbol{u}_{j}: 1 \leq j \leq \ell\right\}$ orthogonal to the vectors $\left\{\hat{\boldsymbol{m}}_{i}: 1 \leq i \leq \theta\right\}$ modulo $g_{i}$ (i.e. a basis of the lattice $\Lambda^{\perp}$, following the previous notation) and we can use this matrix $\boldsymbol{U}$ in order to recover scalar multiples of the plaintext vectors $\left\{\hat{\boldsymbol{m}}_{i}: 1 \leq i \leq \theta\right\}$.

More precisely, we show that for each $1 \leq i \leq \theta$, we can recover the one-dimensional linear space generated by $\hat{\boldsymbol{m}}_{i}$ modulo $g_{i}$. The first step is to factor $g=\prod_{i=1}^{\theta} g_{i}$ to recover the primes $g_{i}$ 's; this is feasible if the $g_{i}$ 's are small enough. ${ }^{1}$ Since we have a basis matrix $\boldsymbol{U}$ of the lattice of vectors $\boldsymbol{u}$

\footnotetext{
${ }^{1}$ For the concrete parameters provided in [CLT13], the $g_{i}$ 's are 80-bit primes; therefore the factorization is straightforward.
} 
with $\left\langle\boldsymbol{u}, \hat{\boldsymbol{m}}_{i}\right\rangle \equiv 0\left(\bmod g_{i}\right)$ for all $1 \leq i \leq \theta$, it suffices to compute the $\mathbb{Z} / g_{i} \mathbb{Z}$-kernel of the $\ell \times \ell$ matrix $\boldsymbol{U}_{g_{i}}=\boldsymbol{U} \bmod g_{i}$; assuming that $\hat{\boldsymbol{m}}_{i} \not \equiv 0\left(\bmod g_{i}\right)$, we have that $\operatorname{ker}\left(\boldsymbol{U}_{g_{i}}\right)$ has dimension 1 over over $\mathbb{Z} / g_{i} \mathbb{Z}$ and therefore, we recover a non-trivial multiple $\lambda_{i} \hat{\boldsymbol{m}}_{i}$ of the original messages $\hat{\boldsymbol{m}}_{i}$ modulo $g_{i}$, for $1 \leq i \leq \theta$. With the ECM [Len87] the factorization of $g=\prod_{i=1}^{\theta} g_{i}$ can be computed in time $\exp (c \sqrt{\alpha \ln \alpha})$ for some positive constant $c$ and where $\alpha$ is the bit size of the $g_{i}$ 's, which gives a sub-exponential time attack.

Alternatively, to avoid the factorization of $g$, we can compute the integer right kernel of the matrix $\left[\boldsymbol{U} \mid g \boldsymbol{I}_{\ell}\right]$, where $\boldsymbol{I}_{\ell}$ denotes the identity matrix in dimension $\ell$. The following proposition shows that we can recover in polynomial time a non-trivial multiple of the vector $\hat{\boldsymbol{m}}$, such that $\hat{\boldsymbol{m}} \equiv \hat{\boldsymbol{m}}_{i}\left(\bmod g_{i}\right)$ for all $1 \leq i \leq \theta$.

Proposition 6. Let $\ell, \theta \in \mathbb{Z}_{\geq 1}$. Let $g_{1}, \ldots, g_{\theta}$ be distinct prime numbers. For $1 \leq i \leq \theta$, let $\hat{\boldsymbol{m}}_{i} \in$ $\mathbb{Z}^{\ell} \cap\left[0, g_{i}\right)^{\ell}$ be vectors such that $\hat{\boldsymbol{m}}_{i} \not \equiv \mathbf{0}\left(\bmod g_{i}\right)$. Let $\left\{\boldsymbol{u}_{j}: 1 \leq j \leq \ell\right\}$ be a basis of the lattice of vectors $\boldsymbol{u} \in \mathbb{Z}^{\ell}$ such that $\left\langle\boldsymbol{u}, \hat{\boldsymbol{m}}_{i}\right\rangle \equiv 0\left(\bmod g_{i}\right)$ for all $1 \leq i \leq \theta$. Then, given $g=\prod_{i=1}^{\theta} g_{i}$ and the vectors $\left\{\boldsymbol{u}_{j}: 1 \leq j \leq \ell\right\}$, one can recover in polynomial time a vector $\lambda \cdot \hat{\boldsymbol{m}} \in \mathbb{Z}^{\ell} \cap[0, g)^{\ell}$ with $\operatorname{gcd}(\lambda, g)=1$, such that $\hat{\boldsymbol{m}} \equiv \hat{\boldsymbol{m}}_{i}\left(\bmod g_{i}\right)$ for all $1 \leq i \leq \theta$.

Proof. By the Chinese Remainder Theorem, there exists a unique vector $\hat{\boldsymbol{m}} \in \mathbb{Z}^{\ell} \cap[0, g)^{\ell}$ satisfying $\hat{\boldsymbol{m}} \equiv \hat{\boldsymbol{m}}_{i}\left(\bmod g_{i}\right)$ for all $1 \leq i \leq \theta$. Consider the composition of maps $\mathbb{Z}^{\ell} \stackrel{\pi}{\rightarrow}(\mathbb{Z} / g \mathbb{Z})^{\ell} \stackrel{\phi}{\rightarrow} \mathbb{Z} / g \mathbb{Z}$, where $\pi$ is reduction modulo $g$ and $\phi$ sends $\boldsymbol{u}$ to $\langle\boldsymbol{u}, \hat{\boldsymbol{m}}\rangle$. By the Chinese Remainder Theorem, the map $\phi$ corresponds to a vector of maps $\phi=\left(\phi_{1}, \ldots, \phi_{\theta}\right):\left(\prod_{i} \mathbb{Z} / g_{i} \mathbb{Z}\right)^{\ell} \rightarrow \prod_{i} \mathbb{Z} / g_{i} \mathbb{Z}$ with components $\phi_{i}:\left(\mathbb{Z} / g_{i} \mathbb{Z}\right)^{\ell} \rightarrow \mathbb{Z} / g_{i} \mathbb{Z}$ for $1 \leq i \leq \theta$. Let $1 \leq i \leq \theta$; since $\hat{\boldsymbol{m}}_{i} \not \equiv \mathbf{0}\left(\bmod g_{i}\right)$, the map $\phi_{i}$ is surjective with kernel $\operatorname{ker}\left(\phi_{i}\right)=\operatorname{im}\left(\boldsymbol{U}_{g_{i}}\right)$ where $\boldsymbol{U}_{g_{i}}=\boldsymbol{U} \bmod g_{i}$. Since $g_{i}$ is prime, $\operatorname{ker}\left(\phi_{i}\right)=\operatorname{im}\left(\boldsymbol{U}_{g_{i}}\right)$ is a $\mathbb{Z} / g_{i} \mathbb{Z}$-vector space of dimension $\ell-1$. It follows that the kernel of $\boldsymbol{U}_{g_{i}}$ has dimension 1 over $\mathbb{Z} / g_{i} \mathbb{Z}$. This holds for all $1 \leq i \leq \theta$, so by the Chinese Remainder Theorem, $\operatorname{ker}\left(\boldsymbol{U}_{g}\right)$ (where $\boldsymbol{U}_{g}$ is the matrix $\boldsymbol{U}$ modulo $g$ ) is a free $\mathbb{Z} / g \mathbb{Z}$-module of rank 1 , generated by $\hat{\boldsymbol{m}}$. In particular, there exists $\boldsymbol{k} \in \mathbb{Z}^{\ell}$ such that $(\hat{\boldsymbol{m}}, \boldsymbol{k})$ belongs to the $\mathbb{Z}$-kernel of the matrix $\left[\boldsymbol{U} \mid g \boldsymbol{I}_{\ell}\right]$. The integer kernel of this matrix can be computed in polynomial time from $g$ and $\boldsymbol{U}$ and the left $\ell \times \ell$ submatrix of the Hermite normal form of the basis of the $\mathbb{Z}$-kernel gives in the first row a vector $\lambda \hat{\boldsymbol{m}}$ with $\lambda \in(\mathbb{Z} / g \mathbb{Z})^{\times}$.

\subsection{Concrete parameters and practical experiments}

Concrete parameters. We provide concrete parameters for CLT13 multilinear maps with independent slots, for various values of the security parameter $\lambda$. We start from the same concrete parameters as provided in [CLT13]; we assume that the encoding noise is set so that the number of extracted bits is $\nu=2 \lambda+12$; we take $\alpha=\lambda$. We then provide the minimum value of $\theta$ that ensures the same level of security against lattice attacks; see Table 1. As in [CLT13], the goal is to ensure that the best attack takes at least $2^{\lambda}$ clock cycles.

While in Table 1 the number of independent slots $n_{\text {slots }}=\lfloor n / \theta\rfloor$ appears to be relatively small, it is always possible to increase the number of independent slots by increasing the value of $n$.

\begin{tabular}{|l|c||c|c|c|c|c|c|}
\hline Instantiation & $\lambda$ & $n$ & $\eta$ & $\gamma=n \cdot \eta$ & $\nu$ & $\theta$ & $n_{\text {slots }}$ \\
\hline \hline Small & 52 & 1080 & 1981 & $2.1 \cdot 10^{6}$ & 116 & 540 & 2 \\
\hline Medium & 62 & 2364 & 2055 & $4.9 \cdot 10^{6}$ & 136 & 1182 & 2 \\
\hline Large & 72 & 8250 & 2261 & $18.7 \cdot 10^{6}$ & 156 & 1472 & 5 \\
\hline Extra & 80 & 26115 & 2438 & $63.7 \cdot 10^{6}$ & 172 & 1789 & 14 \\
\hline
\end{tabular}

Table 1. Concrete parameters for CLT13 multilinear maps with independent slots, for security parameter $\lambda$. 
Practical experiments. We have run our extended attack from Section 4.3 with the "Extra" parameters of CLT13 from Table 1, for increasing values of $\theta$. Note that for such parameters, the original attack from [GLW14] only applies for $\theta=1$. To improve efficiency, we give as input to LLL a truncated matrix basis, where we keep only the $\nu$ most significant bits. Table 2 shows that our attack works in practice for much larger values of $\theta$ than the original attack from [GLW14], which can only work for $\theta=1$. We provide in https://pastebin.com/7WEMHBE9 the source code in Sage $\left[\mathrm{S}^{+} 17\right]$.

\begin{tabular}{|l|c|c|c||c|c|c|}
\cline { 2 - 7 } \multicolumn{1}{c|}{} & $\theta$ & $\alpha$ & $\nu$ & $\ell=d$ & lat. dim. & running time \\
\hline \hline Basic attack [GLW14] & 1 & 80 & 172 & 1 & 2 & $\varepsilon$ \\
\hline Extended attack (Section 4.3) & 2 & 80 & 172 & 2 & 4 & $\varepsilon$ \\
\hline Extended attack (Section 4.3) & 40 & 80 & 172 & 39 & 78 & $10 \mathrm{~s}$ \\
\hline Extended attack (Section 4.3) & 100 & 80 & 172 & 100 & 200 & $11 \mathrm{~min}$ \\
\hline Extended attack (Section 4.3) & 160 & 80 & 172 & 163 & 326 & 11 hours \\
\hline
\end{tabular}

Table 2. Running time of our LLL-based attack, as a function of the parameter $\theta$, for the "Extra" parameters of CLT13. The lattice dimension is $\ell+d=2 \ell$.

\section{Application to the Cheon et al. Attack}

In 2015, Cheon et al. published in $\left[\mathrm{CHL}^{+} 15\right]$ a polynomial time attack against CLT13 resulting in a total break of the multipartite Diffie-Hellman key exchange protocol. The attack relies on the availability of low-level encodings of zero. In this section, we show how to adapt the Cheon et al. attack to the setting of CLT13 with independent slots: we assume that no encodings of zero are available to the attacker (otherwise the Cheon et al. attack would apply immediately), but as previously, the attacker can obtain low-level encodings where only $\theta$ components of the plaintext are non-zero. In particular, this contributes to a cryptanalysis of CLT13 multilinear maps where no encodings of zero are available beforehand; this was considered as an open problem in [CLR15, Section 4].

\subsection{The original Cheon et al. attack with encodings of zero}

We first recall the basic Cheon et al. attack against CLT13. For simplicity, we take $\kappa=3$; the attack is easily extended to $\kappa>3$. Consider a set $\mathcal{A}=\left\{a_{j}: 1 \leq j \leq n\right\}$ of encodings of zero at level one, a pair $\mathcal{B}=\left\{b_{0}, b_{1}\right\}$ of encodings at level one, and a set $\mathcal{C}=\left\{c_{k}: 1 \leq k \leq n\right\}$ of encodings at level one. We write $a_{j} \equiv a_{j i} / z\left(\bmod p_{i}\right), b_{t} \equiv b_{t i} / z\left(\bmod p_{i}\right), c_{k} \equiv c_{k i} / z\left(\bmod p_{i}\right)$, with integers $a_{j i} \equiv 0$ $\left(\bmod g_{i}\right)$, for all $1 \leq j, i, k \leq n$ and $t \in\{0,1\}$. We obtain the zero-testing evaluations:

$$
\omega_{j k}^{(t)}=a_{j} b_{t} c_{k} p_{z t} \bmod x_{0}=\sum_{i=1}^{n} \frac{h_{i}}{g_{i}} a_{j i} b_{t i} c_{k i} \frac{x_{0}}{p_{i}}
$$

where the equality holds over $\mathbb{Z}$ because the products $a_{j} b_{t} c_{k}$ are level-3 encodings of 0 . This can be written in matrix form as

$$
\omega_{j k}^{(t)}=\left[a_{j 1} \cdots a_{j n}\right]\left[\begin{array}{llll}
b_{t 1} p_{z t, 1} & & \\
& \ddots & \\
& & b_{t n} p_{z t, n}
\end{array}\right]\left[\begin{array}{c}
c_{k 1} \\
\vdots \\
c_{k n}
\end{array}\right] .
$$

where $p_{z t, i}=\left(h_{i} / g_{i}\right) \cdot x_{0} / p_{i}$ for all $1 \leq i \leq n$. Writing out the matrices $\boldsymbol{W}_{t}=\left(\omega_{j k}^{(t)}\right)_{1 \leq j, k \leq n}$ for $t \in\{0,1\}$, one obtains the integer matrix equalities $\boldsymbol{W}_{t}=\boldsymbol{A} \Delta_{t} \boldsymbol{C}$ for $t \in\{0,1\}$, where the rows of 
$\boldsymbol{A}$ are the vectors $\left(a_{j 1}, \cdots, a_{j n}\right)_{j}$, the columns of $\boldsymbol{C}$ are the vectors $\left(c_{k 1}, \cdots, c_{k n}\right)_{k}$, and $\Delta_{t}$ is the diagonal matrix $\operatorname{diag}\left(b_{t 1} p_{z t, 1}, \ldots, b_{t n} p_{z t, n}\right)$.

Provided that at least one of $\boldsymbol{W}_{0}, \boldsymbol{W}_{1}$ is invertible over $\mathbb{Q}$ (say $\boldsymbol{W}_{1}$ ), one then evaluates over $\mathbb{Q}$ the matrix product:

$$
\boldsymbol{W}_{0} \cdot \boldsymbol{W}_{1}^{-1}=\boldsymbol{A}\left(\Delta_{0} \Delta_{1}^{-1}\right) \boldsymbol{A}^{-1}
$$

The attacker can thus compute the eigenvalues of $\boldsymbol{W}_{0} \boldsymbol{W}_{1}^{-1}$, by factoring the characteristic polynomial (over $\mathbb{Q}$ ). By similarity of these matrices, these eigenvalues coincide with those of $\Delta_{0} \Delta_{1}^{-1}=$ $\operatorname{diag}\left(b_{01} / b_{11}, \ldots, b_{0 n} / b_{1 n}\right)$, which are $\left\{b_{0 i} / b_{1 i}: 1 \leq i \leq n\right\}$. These ratios are now enough to factor $x_{0}$. Namely, writing the quotients $b_{0 i} / b_{1 i}=x_{i} / y_{i}$ for coprime integers $x_{i}, y_{i}$ and using that $b_{t} \equiv b_{t i} / z$ $\left(\bmod p_{i}\right)$, we obtain:

$$
x_{i} b_{1}-y_{i} b_{0} \equiv\left(x_{i} b_{1 i}-y_{i} b_{0 i}\right) / z \equiv 0 \quad\left(\bmod p_{i}\right)
$$

and therefore $\operatorname{gcd}\left(x_{i} b_{1}-y_{i} b_{0}, x_{0}\right)=p_{i}$ with good probability. In summary, the Cheon et al. attack recovers all secret $p_{i}$ 's in polynomial time given the low-level encodings of zero $\left\{a_{j}: 1 \leq j \leq n\right\}$.

\subsection{Adaptation of the Cheon et al. attack to our cryptanalysis}

We now show how to adapt the Cheon et al. attack when no encodings of zero are available, but the attacker can obtain low-level encodings where only $\theta$ components of the underlying plaintexts are non-zero. The attack is divided in two steps: first the attacker generates encodings of zero using the orthogonal lattice attack from Section 4 , and then applies the original Cheon et al. attack to reveal the primes $\left\{p_{i}: 1 \leq i \leq n\right\}$.

We consider the following setting with $\kappa=4$. Let $\ell \geq 1$; we consider a set $\mathcal{Y}=\left\{y_{j}: 1 \leq j \leq \ell\right\}$ of level-one encodings of messages $\boldsymbol{m}_{1}, \ldots, \boldsymbol{m}_{\ell}$ where only the first $\theta$ components of each $\boldsymbol{m}_{j}$ are nonzero. Moreover, we consider as in the previous section three sets $\mathcal{A}=\left\{a_{j}: 1 \leq j \leq n\right\}, \mathcal{B}=\left\{b_{0}, b_{1}\right\}$ and $\mathcal{C}=\left\{c_{k}: 1 \leq k \leq n\right\}$ of level-one encodings of non-zero messages.

First step: orthogonal lattice attack. We show that the orthogonal lattice attack from Section 4.2 can compute a short vector $\boldsymbol{u} \in \mathbb{Z}^{\ell}$ such that $y^{\prime}=\langle\boldsymbol{u}, \boldsymbol{y}\rangle$ is a level-1 encoding of zero, where $\boldsymbol{y}=\left(y_{1}, \ldots, y_{\ell}\right)$. We write for all $1 \leq j \leq \ell$ :

$$
y_{j} \equiv \frac{r_{j i} \cdot g_{i}+m_{j i}}{z} \quad\left(\bmod p_{i}\right), \quad 1 \leq i \leq n,
$$

with the usual CLT13 notations, where $m_{j i}=0$ for $\theta+1 \leq i \leq n$. Note that our orthogonal lattice attack from Section 4.2 uses level- $\kappa$ encodings; therefore it can be applied on level- $\kappa$ encodings of the form:

$$
e_{j}=y_{j} \cdot a_{1} \cdot b_{0} \cdot c_{1} \bmod x_{0}
$$

for level-one encodings $a_{1}, b_{0}, c_{1}$; we obtain:

$$
e_{j} \equiv \frac{r_{j i}^{\prime} \cdot g_{i}+m_{j i} \cdot x_{i}}{z^{\kappa}} \quad\left(\bmod p_{i}\right), \quad 1 \leq i \leq n
$$

for some $r_{j i}^{\prime} \in \mathbb{Z}$ and where $x_{i}$ is the $i$-th component of the plaintext corresponding to the encoding $a_{1} \cdot b_{0} \cdot c_{1}$. Clearly, since the messages $\left\{\boldsymbol{m}_{j}: 1 \leq j \leq \ell\right\}$ have non-zero support of length $\theta$, the messages $\left\{\left(m_{j i} \cdot x_{i}\right)_{1 \leq i \leq n}: 1 \leq j \leq \ell\right\}$ have non-zero support of length at most $\theta$. Therefore, applying the orthogonal lattice attack from Section 4.2 on the encodings $e_{j}$ (i.e. on the vector $\left.\boldsymbol{\omega}=p_{z t} \cdot\left(e_{j}\right)_{1 \leq j \leq \ell} \bmod x_{0}\right)$, we obtain a vector $\boldsymbol{u} \in \mathbb{Z}^{\ell}$ such that $\left\langle\boldsymbol{u}, \hat{\boldsymbol{m}}_{i} \cdot x_{i}\right\rangle \equiv 0\left(\bmod g_{i}\right)$ for all $1 \leq i \leq \theta$, where the $\hat{\boldsymbol{m}}_{i}$ 's are the vectors $\left(m_{1 i}, \ldots, m_{\ell i}\right)$ for $1 \leq i \leq \theta$. Provided that $x_{i} \not \equiv 0$ 
$\left(\bmod g_{i}\right)$, this implies $\left\langle\boldsymbol{u}, \hat{\boldsymbol{m}}_{i}\right\rangle \equiv 0\left(\bmod g_{i}\right)$ for all $1 \leq i \leq \theta$. Therefore, for all $1 \leq i \leq n$, we can write $\sum_{j=1}^{\ell} u_{j} m_{j i}=k_{i} g_{i}$ for integers $k_{i}$ (and $k_{i}=0$ for $\theta+1 \leq i \leq n$ ). This gives:

$$
y^{\prime}=\sum_{j=1}^{\ell} u_{j} y_{j} \equiv g_{i}\left(\sum_{j=1}^{\ell} u_{j} r_{j i}+k_{i}\right) \cdot z^{-1} \quad\left(\bmod p_{i}\right), \quad 1 \leq i \leq n
$$

and therefore $y^{\prime}$ is a level-1 encoding of zero, moreover with small noise since the vector $\boldsymbol{u}$ is short. Note that we only need a single vector $\boldsymbol{u}$; therefore the first step of the attack is proven by Proposition 5.

Second step: Cheon et al. attack. The second step consists in applying the Cheon et al. attack with the three sets $\mathcal{A}^{\prime}=\left\{y^{\prime} \cdot a_{j}: 1 \leq j \leq n\right\}, \mathcal{B}=\left\{b_{0}, b_{1}\right\}$ and $\mathcal{C}=\left\{c_{k}: 1 \leq k \leq n\right\}$. Since $y^{\prime}$ is an encoding of zero, all encodings in $\mathcal{A}^{\prime}$ are encodings of zero, and we can apply the Cheon et al. attack on the three sets $\mathcal{A}^{\prime}, \mathcal{B}$ and $\mathcal{C}$ to recover all secret primes $p_{i}$.

Since the orthogonal lattice attack more generally provides a set of $\ell$ vectors $\boldsymbol{u}_{j} \in \mathbb{Z}^{\ell}$ (instead of a single $\boldsymbol{u}$; and all satisfying $\left\langle\boldsymbol{u}_{j}, \hat{\boldsymbol{m}}_{i}\right\rangle \equiv 0\left(\bmod g_{i}\right)$ for all $\left.i\right)$, a variant of the above attack with $\kappa=3$ consists in starting from a set $\mathcal{A}=\left\{a_{j}: 1 \leq j \leq n\right\}$ of $\ell=n$ encodings where only the first $\theta$ components of the underlying plaintexts are non-zero, and then generating a set $\mathcal{A}^{\prime}=\left\{\left\langle\boldsymbol{u}_{j}, \boldsymbol{a}\right\rangle: 1 \leq j \leq n\right\}$ of encodings of zero, with the vector of encodings $\boldsymbol{a}=\left(a_{1}, \ldots, a_{n}\right)$. One can then apply the Cheon et al. attack as previously on the three sets $\mathcal{A}^{\prime}, \mathcal{B}$ and $\mathcal{C}$.

Note that the first step of the attack above (i.e. the generation of encodings of zero) uses the orthogonal lattice attack from Section 4.2 with the bound $\alpha \theta<\nu$. The attack from Section 4.3 is easily adapted to reach the improved bound $\alpha \theta=\mathcal{O}\left(\nu^{2}\right)$. In this case the attacker can obtain $\ell \cdot d$ level-two encodings of zero given by $\left\{\left\langle\boldsymbol{u}_{j}, \boldsymbol{c}_{k}\right\rangle: 1 \leq j \leq \ell, 1 \leq k \leq d\right\}$ where $\boldsymbol{c}_{k}$ is the vector of encodings $\left(c_{j} \cdot c_{k}^{\prime}\right)_{1 \leq j \leq \ell}$ with the encodings $c_{j} \cdot c_{k}^{\prime}$ considered in Section 4.3.

\section{Application to constructions based on CLT13 with independent slots}

In this section we show that our orthogonal lattice attack from Section 4 can be applied to various constructions over CLT13 multilinear maps with independent slots.

\subsection{The multilinear subgroup elimination assumption from [GLW14,GLSW15]}

The multilinear subgroup elimination assumption is used in [GLW14] for witness encryption and in [GLSW15] for constructing program obfuscation, based on a single assumption, independent of the particular circuit to be obfuscated. The multilinear subgroup elimination assumption is stated for a generic model of composite-order multilinear maps. Below, we show that our attacks break this assumption over CLT13 composite-order multilinear maps. We note that since the GLW14 scheme also includes encodings of zeroes, it could also be broken more directly by the Cheon et al. attack. We recall the definition from [GLSW15].

Definition $7((\mu, \nu)$-multilinear subgroup elimination assumption [GLSW15]). Let $G$ be a group of order $N=a_{1} \cdots a_{\mu} b_{1} \cdots b_{\nu} c$ where $a_{1}, \ldots, a_{\mu}, b_{1}, \ldots, b_{\nu}, c$ are $\mu+\nu+1$ distinct primes. We give out generators $x_{a_{1}}, \ldots, x_{a_{\mu}}, x_{b_{1}}, \ldots, x_{b_{\nu}}$ for each prime order subgroup except for the subgroup of order $c$. For each $1 \leq i \leq \mu$, we also give out a group element $h_{i}$ sampled uniformly at random from the subgroup of order $c a_{1} \cdots a_{i-1} a_{i+1} \cdots a_{\mu}$. The challenge term is a group element $T \in G$ that is either sampled uniformly at random from the subgroup of order $c a_{1} \cdots a_{\mu}$ or uniformly at random from the subgroup of order $a_{1} \cdots a_{\mu}$. The task is to distinguish between these two distributions of $T$. 
For simplicity, we consider the assumption with $\mu=1$ and $\nu=0$; the generalization of our attack to any $(\mu, \nu)$ is straightforward. Therefore $G$ is a group of order $a_{1} c$. The challenge $T \in G$ is either generated at random from the subgroup of order $a_{1} c$, or from the subgroup of order $a_{1}$. In the context of a CLT13 instantiation, we assume that $a_{1}=\prod_{i=1}^{\theta} g_{i}$ and $c=\prod_{i=\theta+1}^{n} g_{i}$. In that case, $a_{1}$ and $c$ are not primes, but the assumption can still be considered for composite $a_{i}$ 's, $b_{i}$ 's and $c$. The encoding $T$ is then either generated from a random plaintext $m \in \bigoplus_{i=1}^{n} \mathbb{Z} / g_{i} \mathbb{Z}$, or from a random plaintext with only the $\theta$ first components non-zero, that is $m \equiv 0\left(\bmod g_{i}\right)$ for $\theta+1 \leq i \leq n$. It is easy to see that our attacks from Section 4.2 and Section 4.3 apply in this setting. Namely, when only the first $\theta$ components of the plaintext $m$ corresponding to the challenge $T$ are non-zero, our attacks recover the product $a_{1}=\prod_{i=1}^{\theta} g_{i}$, whereas the attacks will fail when $m$ is a random plaintext. Therefore the challenge $T$ is easily distinguished unless $\theta$ is large enough; more precisely, $\theta$ must satisfy the bound given by (23) to prevent the attack.

\subsection{The Zimmerman circuit obfuscation scheme}

At Eurocrypt 2015, Zimmerman described a technique to obfuscate programs without matrix branching programs, based on composite-order multilinear maps [Zim15]. A plaintext $m$ belongs to $\mathbb{Z} / N \mathbb{Z}$ for a composite modulus $N=N_{\mathrm{ev}} \cdot N_{\text {chk }}$, and the ring $\mathbb{Z} / N \mathbb{Z}$ is viewed as a direct product of an "evaluation" ring $\mathbb{Z} / N_{\mathrm{ev}} \mathbb{Z}$ to evaluate the circuit, and a "checksum" ring $\mathbb{Z} / N_{\text {chk }} \mathbb{Z}$ to prevent the adversary from evaluating a different circuit; those two evaluations are performed in parallel. Using the CLT13 notations from Section 2, one can let $N_{\mathrm{ev}}=\prod_{i=1}^{\theta} g_{i}$ and $N_{\mathrm{chk}}=\prod_{i=\theta+1}^{n} g_{i}$. In that case, the parameter $\theta$ must satisfy the bound given by (23) to prevent our lattice attack.

\subsection{The FRS17 construction for preventing input partitioning attacks}

At Asiacrypt 2017, Fernando, Rasmussen and Sahai described three constructions of "stamping functions" for preventing input-partitioning attacks on matrix branching programs [FRS17]. Their third construction is based on permutation hash functions and is instantiated over CLT13 multilinear maps with independent slots. More precisely, the permutation hash function is written as a matrix branching program, and multiple such permutation hash functions $h_{i}$ are evaluated in parallel along with the main matrix branching program; this is to ensure that only inputs of the form $x \| h(x)$ can be evaluated, where $h(x)=h_{1}(x)\|\cdots\| h_{t}(x)$, which prevents input partitioning attacks.

Matrix branching programs. We first recall the construction of $\left[\mathrm{GGH}^{+} 13 \mathrm{~b}\right]$ to obfuscate matrix branching programs. A matrix branching program BP of length $n_{p}$ on $\ell$-bit inputs $x \in\{0,1\}^{\ell}$ is evaluated by computing:

$$
C(x)=\boldsymbol{b}_{0} \cdot \prod_{i=1}^{n_{p}} \boldsymbol{B}_{i, x_{\text {in }(i)}} \cdot \boldsymbol{b}_{n_{p}+1}
$$

where $\left\{\boldsymbol{B}_{i, b}: 1 \leq i \leq n_{p}, b \in\{0,1\}\right\}$ are $2 n_{p}$ square matrices and $\boldsymbol{b}_{0}$ and $\boldsymbol{b}_{n_{p}+1}$ are bookend vectors; then $\operatorname{BP}(x)=0$ if $C(x)=0$, and $\operatorname{BP}(x)=1$ otherwise. The integer inp $(i) \in\{1, \ldots, \ell\}$ indicates which bit of $x$ is read at step $i$ of the product matrix computation. The matrices $\boldsymbol{B}_{i, b}$ are first randomized by choosing $n_{p}+1$ random invertible matrices $\left\{\boldsymbol{R}_{i}: 0 \leq i \leq n_{p}\right\}$ and letting $\tilde{\boldsymbol{B}}_{i, b}=\boldsymbol{R}_{i-1} \boldsymbol{B}_{i, b} \boldsymbol{R}_{i}^{-1}$ for $1 \leq i \leq n_{p}$, with also $\tilde{\boldsymbol{b}}_{0}=\boldsymbol{b}_{0} \boldsymbol{R}_{0}^{-1}$ and $\tilde{\boldsymbol{b}}_{n_{p}+1}=\boldsymbol{R}_{n_{p}} \boldsymbol{b}_{n_{p}+1}$. We obtain a randomized matrix branching program with the same result since the randomization matrices $\boldsymbol{R}_{i}$ cancel each other:

$$
C(x)=\tilde{\boldsymbol{b}}_{0} \cdot \prod_{i=1}^{n_{p}} \tilde{\boldsymbol{B}}_{i, x_{\mathrm{inp}(i)}} \cdot \tilde{\boldsymbol{b}}_{n_{p}+1} .
$$

The entries of the matrices $\tilde{\boldsymbol{B}}_{i, b}$ are then independently encoded, as well as the bookend vectors $\tilde{\boldsymbol{b}}_{0}$ and $\tilde{\boldsymbol{b}}_{n_{p}}$. We obtain the matrices and vectors $\hat{\boldsymbol{B}}_{i, b}=\operatorname{Encode}_{\{i+1\}}\left(\tilde{\boldsymbol{B}}_{i, b}\right), \hat{\boldsymbol{b}}_{0}=\operatorname{Encode}_{\{1\}}\left(\tilde{\boldsymbol{b}}_{0}\right)$ and 
$\hat{\boldsymbol{b}}_{n_{p}+1}=\operatorname{Encode}_{\left\{n_{p}+2\right\}}\left(\tilde{\boldsymbol{b}}_{n_{p}+1}\right)$. Here $\operatorname{Encode}_{\{i\}}(\cdot)$ denotes an encoding relative to the singleton $i$. The matrix branching program from (24) can then be evaluated over the encoded matrices:

$$
\hat{C}(x)=\hat{\boldsymbol{b}}_{0} \cdot \prod_{i=1}^{n_{p}} \hat{\boldsymbol{B}}_{i, x_{\mathrm{inp}(i)}} \cdot \hat{\boldsymbol{b}}_{n_{p}+1}
$$

The resulting $\hat{C}(x)$ is then a last-level encoding that can be zero-tested to check if $C(x)=0$, which reveals the output of the branching program $\operatorname{BP}(x)$, without revealing the matrices $\boldsymbol{B}_{i, b}$.

Application to the FRS17 construction. The [FRS17] scheme constructs a modified matrix branching program $\mathrm{BP}^{\prime}$ that receives as input $u \| v_{1} \ldots v_{t}$ and checks whether $v_{i}=h_{i}(u)$ for all $1 \leq i \leq t$, where the $h_{i}$ 's are permutation hash functions; in that case, $\mathrm{BP}^{\prime}$ returns $\mathrm{BP}(u)$ where $\mathrm{BP}$ is the original branching program; otherwise, it returns some non-zero value. As explained in [FRS17], multiple branching programs can be evaluated in parallel with composite order multilinear maps; with the countermeasure from [GLW14] over CLT13, each branching program is then evaluated modulo a product of $\theta$ of the primes $g_{i}$ 's, instead of a single $g_{i}$ in [FRS17].

It is easy to generate an input $u \| v_{1} \ldots v_{t}$ such that $\operatorname{BP}(u)=0$ and $v_{i}=h_{i}(u)$ for all $1 \leq i \leq t$ except for some $i=i^{\star}$; in that case, only one of the $t+1$ parallel matrix branching program will evaluate to a non-zero value. The orthogonal lattice attack from Section 4.2 can therefore recover the secret plaintext ring $\bigoplus_{i=1}^{n} \mathbb{Z} / g_{i} \mathbb{Z}$ of CLT13, under the condition $\alpha \theta<\nu$. Alternatively, if multiple $p_{z t}$ 's are available, the extended attack from Section 4.3 applies under the condition $\alpha \theta=\mathcal{O}\left(\nu^{2}\right)$, as described at the end of Section 4.3.

We note however that in both cases, our attack against [FRS17] only recovers the secret plaintext ring $\bigoplus_{i=1}^{n} \mathbb{Z} / g_{i} \mathbb{Z}$ of CLT13, and not all secret parameters of CLT13; we leave that as an open problem.

\section{Acknowledgments}

We would like to thank the Asiacrypt 2019 referees for their numerous helpful comments.

\section{References}

AB15. Benny Applebaum and Zvika Brakerski. Obfuscating Circuits via Composite-Order Graded Encoding, pages 528-556. Springer Berlin Heidelberg, Berlin, Heidelberg, 2015.

$\mathrm{CGH}^{+}$15. Jean-Sébastien Coron, Craig Gentry, Shai Halevi, Tancrède Lepoint, Hemanta K. Maji, Eric Miles, Mariana Raykova, Amit Sahai, and Mehdi Tibouchi. Zeroizing without low-level zeroes: New MMAP attacks and their limitations. In CRYPTO 2015, Part I, volume 9215 of LNCS, pages 247-266. Springer, 2015.

CGH17. Yilei Chen, Craig Gentry, and Shai Halevi. Cryptanalyses of candidate branching program obfuscators. In Advances in Cryptology - EUROCRYPT 2017 - Proceedings, Part III, pages 278-307, 2017.

$\mathrm{CHL}^{+}$15. Jung Hee Cheon, Kyoohyung Han, Changmin Lee, Hansol Ryu, and Damien Stehlé. Cryptanalysis of the multilinear map over the integers. In EUROCRYPT 2015, Part I, volume 9056 of LNCS, pages 3-12. Springer, 2015.

CLLT16. Jean-Sébastien Coron, Moon Sung Lee, Tancrède Lepoint, and Mehdi Tibouchi. Cryptanalysis of GGH15 multilinear maps. In Advances in Cryptology - CRYPTO 2016 - Proceedings, Part II, pages 607-628, 2016.

CLLT17. Jean-Sébastien Coron, Moon Sung Lee, Tancrède Lepoint, and Mehdi Tibouchi. Zeroizing attacks on indistinguishability obfuscation over CLT13. In Public-Key Cryptography - PKC 2017 - Proceedings, Part I, pages $41-58,2017$.

CLR15. Jung Hee Cheon, Changmin Lee, and Hansol Ryu. Cryptanalysis of the new CLT multilinear maps. IACR Cryptology ePrint Archive, 2015:934, 2015.

CLT13. Jean-Sébastien Coron, Tancrède Lepoint, and Mehdi Tibouchi. Practical multilinear maps over the integers. In Ran Canetti and Juan A. Garay, editors, CRYPTO, volume 8042 of LNCS, pages 476-493. Springer, 2013.

CVW18. Yilei Chen, Vinod Vaikuntanathan, and Hoeteck Wee. GGH15 beyond permutation branching programs: Proofs, attacks, and candidates. In Advances in Cryptology - CRYPTO 2018 - Proceedings, Part II, pages 577-607, 2018. 
DGHV10. Marten van Dijk, Craig Gentry, Shai Halevi, and Vinod Vaikuntanathan. Fully homomorphic encryption over the integers. In Henri Gilbert, editor, Advances in Cryptology - EUROCRYPT 2010, volume 6110 of Lecture Notes in Computer Science, pages 24-43. Springer, 2010.

FRS17. Rex Fernando, Peter M. R. Rasmussen, and Amit Sahai. Preventing CLT attacks on obfuscation with linear overhead. In Advances in Cryptology - ASIACRYPT 2017 - 23rd International Conference on the Theory and Applications of Cryptology and Information Security, Hong Kong, China, December 3-7, 2017, Proceedings, Part III, pages 242-271, 2017.

GGH13a. Sanjam Garg, Craig Gentry, and Shai Halevi. Candidate multilinear maps from ideal lattices. In Thomas Johansson and Phong Q. Nguyen, editors, EUROCRYPT, volume 7881 of LNCS, pages 1-17. Springer, 2013.

GGH $^{+}$13b. Sanjam Garg, Craig Gentry, Shai Halevi, Mariana Raykova, Amit Sahai, and Brent Waters. Candidate indistinguishability obfuscation and functional encryption for all circuits. In FOCS, pages 40-49. IEEE Computer Society, 2013.

GGH15. Craig Gentry, Sergey Gorbunov, and Shai Halevi. Graph-induced multilinear maps from lattices. In Yevgeniy Dodis and Jesper Buus Nielsen, editors, TCC, volume 9015 of LNCS, pages 498-527, 2015.

GLSW15. Craig Gentry, Allison Bishop Lewko, Amit Sahai, and Brent Waters. Indistinguishability obfuscation from the multilinear subgroup elimination assumption. In IEEE 56th Annual Symposium on Foundations of Computer Science, FOCS 2015, Berkeley, CA, USA, 17-20 October, 2015, pages 151-170, 2015.

GLW14. Craig Gentry, Allison B. Lewko, and Brent Waters. Witness encryption from instance independent assumptions. In Advances in Cryptology - CRYPTO 2014 - 34th Annual Cryptology Conference, Santa Barbara, CA, USA, August 17-21, 2014, Proceedings, Part I, pages 426-443, 2014. https://eprint. iacr.org/2014/273.

HJ16. Yupu Hu and Huiwen Jia. Cryptanalysis of GGH map. In Advances in Cryptology - EUROCRYPT 2016 - Proceedings, Part I, pages 537-565, 2016.

HPS11. Guillaume Hanrot, Xavier Pujol, and Damien Stehlé. Analyzing blockwise lattice algorithms using dynamical systems. In Proceedings of the 31st Annual Conference on Advances in Cryptology, CRYPTO'11, pages 447-464, Berlin, Heidelberg, 2011. Springer-Verlag.

Len87. H. W. Lenstra, Jr. Factoring integers with elliptic curves. Ann. of Math. (2), 126(3):649-673, 1987.

LLL82. A. K. Lenstra, H. W. Lenstra, and L. Lovasz. Factoring polynomials with rational coefficients, 1982.

MSZ16. Eric Miles, Amit Sahai, and Mark Zhandry. Annihilation attacks for multilinear maps: Cryptanalysis of indistinguishability obfuscation over GGH13. In Advances in Cryptology - CRYPTO 2016 - Proceedings, Part II, pages 629-658, 2016.

NS99. Phong Nguyen and Jacques Stern. The hardness of the hidden subset sum problem and its cryptographic implications, 1999.

$\mathrm{S}^{+}$17. W. A. Stein et al. Sage Mathematics Software (Version 8.0). The Sage Development Team, 2017. http://www . sagemath.org.

Zim15. Joe Zimmerman. How to obfuscate programs directly. In Advances in Cryptology - EUROCRYPT 2015 - 34th Annual International Conference on the Theory and Applications of Cryptographic Techniques, Sofia, Bulgaria, April 26-30, 2015, Proceedings, Part II, pages 439-467, 2015.

\section{A Proofs}

\section{A.1 Proof of Lemma 4}

Let $\mathcal{B}=\left\{\left(B \boldsymbol{u}_{j}, v_{j}\right): 1 \leq j \leq \ell+1\right\}$ be a basis of $L$. We show that the vectors $\left\{\boldsymbol{u}_{j}: 1 \leq j \leq \ell\right\}$ corresponding to the first $\ell$ vectors, must necessarily be linearly independent over $\mathbb{R}$. For the sake of contradiction, we assume they are linearly dependent. For every vector $\left(B \boldsymbol{u}_{j}, v_{j}\right)($ with $1 \leq j \leq \ell)$, we consider the associated vector $\boldsymbol{p}_{\boldsymbol{u}_{j}, v_{j}}$. By assumption, the vectors $\left\{\boldsymbol{p}_{\boldsymbol{u}_{j}, v_{j}}: 1 \leq j \leq \ell\right\}$ belong to the lattice generated by the vectors $\left\{\boldsymbol{q}_{i}: 1 \leq i \leq \theta\right\}$, so there are integers $\beta_{i j} \in \mathbb{Z}$ such that $\boldsymbol{p}_{\boldsymbol{u}_{j}, v_{j}}=\sum_{i=1}^{\theta} \beta_{i j} \boldsymbol{q}_{i}$ for every $1 \leq j \leq \ell$. The definition of the vectors $\left\{\boldsymbol{q}_{i}: 1 \leq i \leq \theta\right\}$ gives $\boldsymbol{p}_{\boldsymbol{u}_{j}, v_{j}}=\left(C \beta_{1 j} g_{1}, \ldots, C \beta_{\theta j} g_{\theta},-\sum_{i=1}^{\theta} \beta_{i j} s_{i}\right)$ for every $1 \leq j \leq \ell$; and from the definition of the vector $\boldsymbol{p}_{\boldsymbol{u}_{j}, v_{j}}$, we conclude by equalizing the components, the relations

$$
\beta_{i j} g_{i}=\left\langle\boldsymbol{u}_{j}, \hat{\boldsymbol{m}}_{i}\right\rangle
$$

and

$$
-\sum_{i=1}^{\theta} \beta_{i j} s_{i}=\left\langle\boldsymbol{u}_{j}, \boldsymbol{R}\right\rangle+v_{j}
$$


for every $1 \leq j \leq \ell, 1 \leq i \leq \theta$. Combining Equations (26) and (27) gives

$$
v_{j}=-\sum_{i=1}^{\theta} \frac{s_{i}}{g_{i}}\left\langle\boldsymbol{u}_{j}, \hat{\boldsymbol{m}}_{i}\right\rangle-\left\langle\boldsymbol{u}_{j}, \boldsymbol{R}\right\rangle, 1 \leq j \leq \ell
$$

This implies that if the vectors $\left\{\boldsymbol{u}_{j}: 1 \leq j \leq \ell\right\}$ are linearly dependent over $\mathbb{R}$, then also the vectors $\left\{\left(B \boldsymbol{u}_{j}, v_{j}\right): 1 \leq j \leq \ell\right\}$ are linearly dependent over $\mathbb{R}$, which contradicts the fact that $\mathcal{B}$ is a basis of $L$.

\section{A.2 Proof of Proposition 5}

Let $\boldsymbol{a}=\left(\alpha_{1}, \ldots, \alpha_{\theta}, 1\right) \in \mathbb{Z}^{\theta+1}$. We let $C=2^{\rho_{R}-\alpha+1}$ and consider the lattice $A^{\perp}$ of vectors $(C \boldsymbol{x}, y) \in$ $\mathbb{Z}^{\theta} \times \mathbb{Z}$ such that $(\boldsymbol{x}, y)$ is orthogonal to $\boldsymbol{a}$ modulo $x_{0}$. Further, we let $B=\theta 2^{\rho_{R}+2}$ and let $L \subseteq \mathbb{Z}^{\ell+1}$ denote the lattice of vectors $(B \boldsymbol{u}, v) \in \mathbb{Z}^{\ell} \times \mathbb{Z}$ such that the vector $(\boldsymbol{u}, v)$ is orthogonal to the vector $(\boldsymbol{\omega}, 1)$ modulo $x_{0}$.

Let $\Lambda^{\perp}$ be the lattice of vectors $\boldsymbol{u} \in \mathbb{Z}^{\ell}$ such that $\left\langle\boldsymbol{u}, \hat{\boldsymbol{m}}_{i}\right\rangle \equiv 0\left(\bmod g_{i}\right)$ for all $1 \leq i \leq \theta$. We denote by $\boldsymbol{u}_{0}$ a shortest non-zero vector of $\Lambda^{\perp}$. We write $\left\langle\boldsymbol{u}_{0}, \hat{\boldsymbol{m}}_{i}\right\rangle=k_{i} g_{i}$ with $k_{i} \in \mathbb{Z}$. To $\boldsymbol{u}_{0}$ we thus associate the vector $F\left(\boldsymbol{u}_{0}\right)=\left(B \boldsymbol{u}_{0},-\sum_{i=1}^{\theta} k_{i} s_{i}-\left\langle\boldsymbol{u}_{0}, \boldsymbol{R}\right\rangle\right)$. From the definition of $\boldsymbol{\omega}$ and the congruence relations $g_{i} \alpha_{i} \equiv s_{i}\left(\bmod x_{0}\right)$, we have that $\left(\boldsymbol{u}_{0},-\sum_{i=1}^{\theta} k_{i} s_{i}-\left\langle\boldsymbol{u}_{0}, \boldsymbol{R}\right\rangle\right)$ is orthogonal to $(\boldsymbol{\omega}, 1)$ modulo $x_{0}$, and therefore $F\left(\boldsymbol{u}_{0}\right) \in L$.

Letting $g=\prod_{i=1}^{\theta} g_{i}$, we now show that $F\left(\boldsymbol{u}_{0}\right)$ has square norm upper bounded by

$$
\left\|F\left(\boldsymbol{u}_{0}\right)\right\|^{2} \leq(\ell+1) B^{2}\left\|\boldsymbol{u}_{0}\right\|^{2} \leq \ell(\ell+1) B^{2} g^{2 / \ell} .
$$

Indeed, we write $\left\|F\left(\boldsymbol{u}_{0}\right)\right\|^{2} \leq B^{2}\left\|\boldsymbol{u}_{0}\right\|^{2}+\left(\sum_{i=1}^{\theta}\left|k_{i} s_{i}\right|+\left\|\boldsymbol{u}_{0}\right\|\|\boldsymbol{R}\|\right)^{2}$. From $\left\|\hat{\boldsymbol{m}}_{i}\right\| \leq \sqrt{\ell} 2^{\alpha}$, we obtain $2^{\alpha-1}\left|k_{i}\right| \leq\left|k_{i}\right| g_{i} \leq\left\|\boldsymbol{u}_{0}\right\|\left\|\hat{\boldsymbol{m}}_{i}\right\| \leq \sqrt{\ell} 2^{\alpha}\left\|\boldsymbol{u}_{0}\right\|$; i.e. $\left|k_{i}\right| \leq 2 \sqrt{\ell}\left\|\boldsymbol{u}_{0}\right\|$ for all $i$. Combined with $\|\boldsymbol{R}\| \leq$ $\sqrt{\ell}\|\boldsymbol{R}\|_{\infty} \leq \sqrt{\ell} 2^{\rho_{R}}$, this gives

$$
\sum_{i=1}^{\theta}\left|k_{i} s_{i}\right|+\left\|\boldsymbol{u}_{0}\right\|\|\boldsymbol{R}\| \leq \sqrt{\ell}\left\|\boldsymbol{u}_{0}\right\| \cdot\left(2^{\rho_{R}+1} \theta+2^{\rho_{R}}\right) \leq \sqrt{\ell}\left\|\boldsymbol{u}_{0}\right\|\left(2 \cdot 2^{\rho_{R}+1} \theta\right)=\sqrt{\ell} B\left\|\boldsymbol{u}_{0}\right\|
$$

Therefore, $\left\|F\left(\boldsymbol{u}_{0}\right)\right\|^{2} \leq B^{2}\left\|\boldsymbol{u}_{0}\right\|^{2}+\ell B^{2}\left\|\boldsymbol{u}_{0}\right\|^{2}=(\ell+1) B^{2}\left\|\boldsymbol{u}_{0}\right\|^{2}$. Now, since $\boldsymbol{u}_{0}$ has length $\lambda_{1}\left(\Lambda^{\perp}\right)$, it follows from Minkowski's Theorem that $\left\|\boldsymbol{u}_{0}\right\| \leq \sqrt{\ell} g^{1 / \ell}$ where $g=\operatorname{det}\left(\Lambda^{\perp}\right)$, and (28) easily follows.

Let $\boldsymbol{x}_{1}=\left(B \boldsymbol{u}_{1}, v_{1}\right)$ be the first vector in a $(3 / 4)$-reduced basis of the lattice $L$, obtained from LLL. By Theorem 3, it satisfies $\left\|\boldsymbol{x}_{1}\right\| \leq 2^{\ell / 2}\left\|F\left(\boldsymbol{u}_{0}\right)\right\|$, that is, combined with (28), $\left\|\boldsymbol{x}_{1}\right\| \leq$ $2^{\ell / 2} \sqrt{\ell(\ell+1)} B g^{1 / \ell}$. In particular, we obtain the bounds

$$
\begin{array}{r}
\left\|\boldsymbol{u}_{1}\right\| \leq 2^{\ell / 2} \sqrt{\ell(\ell+1)} \cdot g^{1 / \ell} \\
\left|v_{1}\right| \leq 2^{\ell / 2} B \sqrt{\ell(\ell+1)} \cdot g^{1 / \ell} .
\end{array}
$$

For simplicity we write $K=2^{\ell / 2} \sqrt{\ell(\ell+1)} g^{1 / \ell}$. Now, to the vector $\boldsymbol{x}_{1} \in L$, we associate, for $C$ as above, the vector $f\left(\boldsymbol{x}_{1}\right)=\left(C\left\langle\boldsymbol{u}_{1}, \hat{\boldsymbol{m}}_{1}\right\rangle, \ldots, C\left\langle\boldsymbol{u}_{1}, \hat{\boldsymbol{m}}_{\theta}\right\rangle,\left\langle\boldsymbol{u}_{1}, \boldsymbol{R}\right\rangle+v_{1}\right) \in A^{\perp}$. Because $\left(B \boldsymbol{u}_{1}, v_{1}\right) \in L$, it is a direct check that $f\left(\boldsymbol{x}_{1}\right) \in A^{\perp}$. Its square norm is upper bounded by

$$
\left\|f\left(\boldsymbol{x}_{1}\right)\right\|^{2} \leq C^{2} \sum_{i=1}^{\theta}\left\|\boldsymbol{u}_{1}\right\|^{2}\left\|\hat{\boldsymbol{m}}_{i}\right\|^{2}+\left(\left\|\boldsymbol{u}_{1}\right\|\|\boldsymbol{R}\|+v_{1}\right)^{2} .
$$

Using once again that $\left\|\hat{\boldsymbol{m}}_{i}\right\| \leq 2^{\alpha} \sqrt{\ell}$ and $\|\boldsymbol{R}\| \leq 2^{\rho_{R}} \sqrt{\ell}$, and combining with (29) and (30), we obtain

$$
\begin{aligned}
\left\|f\left(\boldsymbol{x}_{1}\right)\right\|^{2} & \leq C^{2} K^{2} \cdot \theta \ell 2^{2 \alpha}+\left(K \sqrt{\ell} 2^{\rho_{R}}+K B\right)^{2} \leq C^{2} K^{2} \cdot \theta \ell 2^{2 \alpha}+(2 K \sqrt{\ell} B)^{2} \\
& =K^{2} \ell\left(C^{2} \theta 2^{2 \alpha}+4 B^{2}\right)
\end{aligned}
$$


so that, using $C^{2} \theta 2^{2 \alpha} \leq B^{2}=16 \theta^{2} 2^{2 \rho_{R}}$, this gives

$$
\left\|f\left(\boldsymbol{x}_{1}\right)\right\| \leq 4 \sqrt{5} \cdot \sqrt{\ell} \cdot \theta \cdot K \cdot 2^{\rho_{R}} .
$$

We now consider the vectors $\left\{\boldsymbol{q}_{i}: 1 \leq i \leq \theta\right\}$ defined by $\boldsymbol{q}_{i}=\left(0, \ldots 0, C g_{i}, 0, \ldots, 0,-s_{i}\right) \in \mathbb{Z}^{\theta+1}$. They are linearly independent; moreover, from the congruence relations $g_{i} \alpha_{i} \equiv s_{i}\left(\bmod x_{0}\right)$ for $1 \leq$ $i \leq \theta$ we deduce that for all $i,\left\langle\boldsymbol{q}_{i}, \boldsymbol{a}\right\rangle \equiv 0\left(\bmod x_{0}\right)$; i.e. $\boldsymbol{q}_{i} \in A^{\perp}$. Further, as $\left|s_{i}\right| \leq 2^{\rho_{R}}$, their norm is upper bounded by $\left\|\boldsymbol{q}_{i}\right\|^{2} \leq C^{2} g_{i}^{2}+2^{2 \rho_{R}} \leq C^{2} g_{i}^{2}+C g_{i}^{2} \leq 2 C^{2} g_{i}^{2}$ because $C g_{i} \geq 2^{\rho_{R}-\alpha+1} \cdot 2^{\alpha-1}=2^{\rho_{R}}$. Consequently,

$$
\prod_{i=1}^{\theta}\left\|\boldsymbol{q}_{i}\right\| \leq 2^{\theta / 2} C^{\theta} \prod_{i=1}^{\theta} g_{i}=2^{\theta / 2} C^{\theta} g .
$$

Now, (17) together with $g \leq 2^{\alpha \theta}$, implies $(1+1 / \ell) \log _{2}(g)+(\ell+\theta) / 2+\log _{2}(4 \sqrt{5} \sqrt{\ell+1} \theta \ell)<$ $\log _{2}\left(x_{0}\right)-\rho_{R}$ and, by raising to the power of 2 , we obtain $g^{1+1 / \ell} \cdot 2^{\ell / 2} \cdot 2^{\theta / 2} \cdot 4 \sqrt{5} \sqrt{\ell+1} \theta \ell<x_{0} / 2^{\rho_{R}}$. This is equivalent to

$$
g^{1 / \ell} \cdot 2^{\ell / 2} \cdot 2^{\rho_{R}} \cdot 4 \sqrt{5} \sqrt{\ell+1} \cdot \theta \ell<\frac{C^{\theta} x_{0}}{C^{\theta} 2^{\theta / 2} g} .
$$

The left hand side is lower bounded by $\left\|f\left(\boldsymbol{x}_{1}\right)\right\|$ by (31), and the right hand side is upper bounded by $\operatorname{det}\left(A^{\perp}\right) / \prod_{i=1}^{\theta}\left\|\boldsymbol{q}_{i}\right\|$, by (32) together with $\operatorname{det}\left(A^{\perp}\right)=C^{\theta} x_{0}$. Therefore (33) implies $\left\|f\left(\boldsymbol{x}_{1}\right)\right\|<$ $\operatorname{det}\left(A^{\perp}\right) / \prod_{i=1}^{\theta}\left\|\boldsymbol{q}_{i}\right\|$. It follows from Lemma 2 that $f\left(\boldsymbol{x}_{1}\right)$ is in the linear span generated by the vectors $\left\{\boldsymbol{q}_{i}: 1 \leq i \leq \theta\right\}$. Since $g_{i}$ are distinct prime numbers and $\operatorname{gcd}\left(s_{i}, g_{i}\right)=1$ for $1 \leq i \leq \theta$, we conclude that $f\left(\boldsymbol{x}_{1}\right)$ is in the sublattice generated by the vectors $\left\{\boldsymbol{q}_{i}: 1 \leq i \leq \theta\right\}$. Consequently, for all $1 \leq i \leq \theta$, one has $\left\langle\boldsymbol{u}_{1}, \hat{\boldsymbol{m}}_{i}\right\rangle \equiv 0\left(\bmod g_{i}\right)$.

The rows $\left\{\boldsymbol{b}_{j}: 1 \leq j \leq \ell+1\right\}$ of the matrix

$$
\left[\begin{array}{cc}
B \boldsymbol{I}_{\ell}-\boldsymbol{\omega}^{T} \\
0 & x_{0}
\end{array}\right]
$$

where $\boldsymbol{I}_{\ell}$ denotes the $\ell \times \ell$ identity matrix, form a $\mathbb{Z}$-basis of $L$. Hence, by running LLL on this matrix with $\delta=3 / 4$, we obtain a vector $\boldsymbol{x}_{1}$ of which the first $\ell$ entries, divided by $B$, produce a vector $\boldsymbol{u}=\boldsymbol{u}_{1}$ satisfying $\left\langle\boldsymbol{u}_{1}, \hat{\boldsymbol{m}}_{i}\right\rangle \equiv 0\left(\bmod g_{i}\right)$ for all $i$. By Theorem 3 , the algorithm terminates in polynomial time. 